J. Roosen, J. Spooren, K. Binnemans

Journal of Materials Chemistry A 2, 19415-19426 (2014)

\title{
Adsorption Performance of Functionalized Chitosan-Silica Hybrid Materials toward Rare Earths
}

Joris Roosen, ${ }^{\mathrm{a}, \mathrm{b}}$ Jeroen Spooren $^{\mathrm{b}}$ and Koen Binnemans ${ }^{\mathrm{a} *}$

${ }^{a}$ KU Leuven, Department of Chemistry, Celestijnenlaan 200F, P.O. Box 2404, B-3001 Heverlee (Belgium).

${ }^{b}$ VITO, Sustainable Materials Management, Boeretang 200, B-2400 Mol (Belgium).

* Corresponding author:

E-mail: Koen.Binnemans@chem.kuleuven.be

Phone: +32163274 46

Fax: +3216327992 


\section{ABSTRACT}

Chitosan-silica hybrid adsorbents were prepared and functionalized with ethylenediaminetetraacetic acid (EDTA) and diethylenetriaminepentaacetic acid (DTPA). The method consisted of a sol-gel hybridization of chitosan and silica, followed by the addition of anhydrides to graft EDTA- and DTPA-ligands on the amine groups of the chitosan moieties in the hybrid particles. The resulting adsorbents were characterized by a range of analytical techniques: FTIR, BET, SEM, TGA, ICP and CHN. Coordination of Eu(III) to immobilized EDTA- and DTPA-groups was investigated by luminescence spectroscopy. The adsorption performance of the chitosan-silica adsorbents was investigated for $\mathrm{Nd}(\mathrm{III})$ as a function of the contact time, the $\mathrm{pH}$ of the aqueous feed and the adsorbent mass. Adsorption isotherms were obtained by increasing the absorbent mass. Stripping and reusability studies were performed for both EDTA-chitosan-silica and DTPA-chitosan-silica. Differences in affinity amongst the rareearth ions were investigated for DTPA-chitosan-silica in mono-component solutions of five rare earths (La, Nd, Eu, Dy and Lu). The order of affinity was in agreement with the trend in stability constants for the respective rare-earth ions with non-immobilized DTPA (bearing five available carboxylic acid groups). Multi-element mixtures were used to determine the selectivity of the adsorption process. Special attention was paid to $\mathrm{Nd} / \mathrm{Dy}$ separations, since these elements are relevant to the recovery of rare earths from End-of-Life permanent magnets.

Keywords: biosorbents; chitosan; silica; hybrid materials; lanthanides; rare earths 


\section{INTRODUCTION}

Biosorption is a promising technology for the removal or recovery of organic and inorganic substances from solution. ${ }^{1}$ In our evolution towards a more sustainable society, biosorption offers advantages due to the cost-effective, environmentally friendly and virtually unlimited supply of bioresources. ${ }^{2}$ A wide variety of biosorbents exist, ranging from micro-organisms to agricultural waste. ${ }^{3-5}$ One of the most promising biosorbents is chitosan, a linear polysaccharide composed of randomly distributed $\beta$-(1,4)-linked D-glucosamine (deacetylated unit) and $N$ acetyl-D-glucosamine (acetylated unit), which is obtained on an industrial scale by the alkaline deacetylation of chitin. As the main component in the exoskeleton of Crustacea, chitosan is one of the most abundant biopolymers in nature. ${ }^{6,7}$ Besides the non-toxicity, bio-degradability and reusability of biosorbents, chitosan is specifically advantageous because it contains a high concentration of amino groups, which are easy to functionalize. This results in a high adsorption capacity and selectivity for metal ions. ${ }^{8-17}$ Modified chitosan is also very useful as a support material in heterogeneous catalysis. ${ }^{18-22}$ However, chitosan suffers from poor mechanical properties and low chemical resistance. ${ }^{23}$ To improve the properties of chitosan materials for use in metal-ion recovery, chitosan has been modified with ceramic alumina, ${ }^{24}$ alginate, ${ }^{25}$ polyvinyl alcohol,${ }^{26}$ cyclodextrins, ${ }^{27}$ magnetic nanoparticles, ${ }^{28}$ ionic liquids, ${ }^{29}$ and silica. ${ }^{30-36}$ By these modifications, the advantages of multiple materials are combined into one superior material. Combination of chitosan with silica has been shown to be suited as supporting material for column chromatography because of the large surface area, the high porosity and the excellent mechanical resistance of the resulting particles. ${ }^{36}$ The simplest materials have a chitosan coating on the surface of silica particles, whereas the truly hybrid materials are prepared by a sol-gel process with hydrolysis of a silicon alkoxide precursor in the presence of chitosan. The sol-gel process results in the formation of covalent bonds between the chitosan and the silica network. 
Chitosan-silica hybrid materials have been investigated for the adsorption of only a limited number of metals $(\mathrm{Co}, \mathrm{Ni}, \mathrm{Cd}$ and $\mathrm{Pb})$, but it offers many possibilities. ${ }^{34}$ Most studies focused on the removal of unwanted species from waste waters like heavy metals, or charged organic species like cationic dyes. ${ }^{37}$ However, chitosan-silica hybrids could also have great potential as sorbents for the selective recovery of valuable metals from secondary resources. Valorization of industrial waste streams can for instance be interesting in the case of red mud, phosphogypsum and other industrial residues. These specific waste streams can contain significant amounts of rare earths. ${ }^{38}$ Because of their essential role in permanent magnets, catalysts, rechargeable batteries, lamp phosphors, etc., the demand for rare earths will continuously grow in the future. A supply risk for rare-earth elements (REE) exists because of China's quasi-monopoly on the production of rare earths, combined with a strict export policy. The recovery and recycling of rare earths is thus a very important issue. ${ }^{39-41}$ The number of studies on the use of biomass for the adsorption of rare earths is limited. ${ }^{42-44}$ Nevertheless, results up to now confirm the importance of research about the use of biosorbents for the recovery of rare-earth elements.

In this paper, we describe the synthesis and characterization of EDTA- and DTPAfunctionalized chitosan-silica hybrid particles and the application of these materials for the recovery of trivalent rare-earth ions from diluted aqueous solutions. 


\section{EXPERIMENTAL SECTION}

\section{Materials}

For the synthesis of the chitosan-silica hybrid particles, lowly viscous chitosan from shrimp shells ( $\geq 99 \%$ purity) was obtained from Sigma-Aldrich and tetraethyl orthosilicate (TEOS, $\geq$ 99\% pure) from Fluka Chemika. Ammonia solution (Analar Normapur, $25 \mathrm{wt} \%$ ) and hydrogen chloride (ACS reagent, 37\%) were obtained from VWR. Ethanol (Disinfectol®, denaturated with up to $5 \%$ ether) was obtained from Chem-Lab. Deuterium oxide $\left(\mathrm{D}_{2} \mathrm{O}, 99.9\right.$ at. \% $\left.\mathrm{D}\right)$ was purchased from Sigma-Aldrich. Aqueous rare-earth solutions for adsorption experiments were made from their corresponding REE salts: $\mathrm{La}\left(\mathrm{NO}_{3}\right)_{3} \cdot 6 \mathrm{H}_{2} \mathrm{O}(99.9 \%)$ was supplied by Chempur, $\mathrm{Nd}\left(\mathrm{NO}_{3}\right)_{3} \cdot 6 \mathrm{H}_{2} \mathrm{O}(99.9 \%)$ and $\mathrm{Dy}\left(\mathrm{NO}_{3}\right)_{3} \cdot 5 \mathrm{H}_{2} \mathrm{O}(99.9 \%)$ were supplied by Alfa Aesar, $\mathrm{Eu}\left(\mathrm{NO}_{3}\right)_{3} \cdot 6 \mathrm{H}_{2} \mathrm{O}(99.9 \%)$ was supplied by Strem Chemicals and $\mathrm{Lu}\left(\mathrm{NO}_{3}\right)_{3} \cdot \mathrm{xH}_{2} \mathrm{O}$ was supplied by Sigma-Aldrich. Proper dilutions were made with MilliQ ${ }^{\circledR}$ water (Millipore, $>18 \mathrm{M} \Omega \mathrm{cm}^{-1}$ ). A 1000 ppm gallium standard was obtained from Merck. The silicone solution in isopropanol was obtained from SERVA Electrophoresis GmbH. All chemicals were used as received without further purification.

\section{Equipment and analysis}

FTIR spectra were recorded on a Bruker Vertex 70 spectrometer (Bruker Optics). Samples were examined as such using a Platinum ATR single reflection diamond attenuated total reflection (ATR) accessory. Scanning Electron Microscopy (SEM) was performed to investigate the surface morphology. Images were made at an acceleration voltage of $5 \mathrm{kV}$ on a JEOL JSM6340F apparatus equipped with a Bruker X Flash Detector 5030 and a Bruker QUANTAX 200 EDS system. The specific surface area and porosity of the adsorbents were determined with a Quantachrome Autosorb-iQ automated gas sorption analyzer. Samples were outgassed under 
inert helium purge, at a final outgas temperature of $135^{\circ} \mathrm{C}$. The surface area and pore size were derived using the BET method by analyzing nitrogen adsorption at liquid nitrogen temperature. Thermogravimetric analysis (TGA) was performed to determine the organic content using a Netzsch-Gerätebau STA 449 C Jupiter thermo-microbalance which was coupled to a Pfeiffer Vacuum OmniStar mass spectrometer. Samples were analyzed from ambient temperature to $1000{ }^{\circ} \mathrm{C}$ under flowing air at a heating rate of $5{ }^{\circ} \mathrm{C} / \mathrm{min}$. $\mathrm{CHN}$ (carbon, hydrogen, nitrogen) elemental analyses were obtained with the aid of a CE Instruments EA-1110 element analyzer. Inductively Coupled Plasma - Atomic Emission Spectroscopy (ICP-AES) was used to analyze the silicon content in the synthesized particles. Samples were destructed in an Anton Paar Multiwave 3000 microwave after mixing them with a ternary mixture of $\mathrm{HCl} 37 \%(2 \mathrm{~mL}), \mathrm{HF}$ $48 \%(4 \mathrm{~mL})$ and $\mathrm{HNO}_{3} 65 \%(6 \mathrm{~mL})$ in a Teflon disclosure recipient. $\mathrm{HF}$, that could form volatile $\mathrm{SiF}_{4}$ compounds, was then neutralized with $\mathrm{H}_{3} \mathrm{BO}_{3}$. The samples were analyzed with an ICP IRIS Intrepid XUV using the axial $251.611 \mathrm{~nm}$ emission line of silicon. Luminescence spectra and decay curves were recorded at room temperature on an Edinburgh Instruments FS900 spectrofluorimeter, equipped with a $450 \mathrm{~W}$ xenon arc lamp and a $50 \mathrm{~W}$ microsecond xenon flash lamp. Metal ion concentrations were determined by means of total-reflection X-ray fluorescence (TXRF) on a Bruker S2 Picofox TXRF spectrometer. To perform the sample preparation for a TXRF measurement, the unknown metal ion solution $(900 \mu \mathrm{L})$ is mixed in an Eppendorf tube with a $1000 \mathrm{mg} \cdot \mathrm{L}^{-1}$ gallium standard solution $(100 \mu \mathrm{L})$ and stirred. A small amount of this prepared solution (about $7.5 \mu \mathrm{L}$ ) is put on a small quartz plate, pre-coated with a hydrophobic silicone solution (about $10 \mu \mathrm{L}$ ), and dried in an oven at $60^{\circ} \mathrm{C}$. Centrifugation was done by means of a Heraeus Megafuge 1.0 centrifuge. 


\section{Synthesis}

\section{Chitosan-silica (CS)}

The chitosan-silica hybrid materials were synthesized according to the in-situ, Stöber biased method described by Rashidova et al. ${ }^{33}$ Chitosan $(2.0 \mathrm{~g})$ was dissolved first in a 2 vol\% acetic acid solution $(100 \mathrm{~mL})$. TEOS $(30 \mathrm{~mL})$ was added to the pale yellow viscous solution. The solution ( $\mathrm{pH} 4$ ) was stirred for $1 / 2 \mathrm{~h}$ to induce the hydrolysis reactions, during which ethoxide groups are replaced by hydroxyl groups. Then the solution was poured into a flask containing a solution of 3 vol\% $\mathrm{NH}_{3}(200 \mathrm{~mL}, \mathrm{pH} 12)$ to catalyze the condensation reactions. The resulting white suspension ( $\mathrm{pH} 10)$ was strongly stirred for $24 \mathrm{~h}$ at room temperature. The chitosan-silica slurry was filtered off and thoroughly washed with a considerable amount of demineralized water until neutral $\mathrm{pH}$. Then the product was washed with ethanol and $n$-heptane. Eventually it was air-dried for $24 \mathrm{~h}$, before vacuum-drying it at $40{ }^{\circ} \mathrm{C}$ for $24 \mathrm{~h}$. The resulting material was a white powder. Yield: $9.0 \pm 0.5 \mathrm{~g}$.

\section{EDTA-chitosan-silica and DTPA-chitosan-silica}

As described by Repo et al., ${ }^{34}$ the chitosan-silica hybrid materials could further be functionalized with EDTA and DTPA by grafting the corresponding anhydrides (excess) on the chitosan amino groups. The bisanhydride synthesis was fully described in previous work. ${ }^{44}$ The functionalization occurred in a solution of chitosan-silica $(7.5 \mathrm{~g})$, acetic acid $(5 \mathrm{vol} \%, 100 \mathrm{~mL})$ and methanol (400 $\mathrm{mL)}$. Yield: $5.5 \pm 0.5 \mathrm{~g}$.

\section{Adsorption}

Batch adsorption experiments were first carried out with aqueous solutions of neodymium(III) to characterize several adsorption parameters: influence of contact time, $\mathrm{pH}$ of the aqueous feed and 
adsorbent mass. The $\mathrm{Nd}^{3+}$ ion was used as a model system for all rare-earth ions in the optimization tests. All adsorption tests were performed in a $10 \mathrm{~mL}$ aliquot of a properly diluted stock solution. The adsorbent $(25.0 \pm 0.1 \mathrm{mg})$ was added to the vials. Solutions were then stirred at room temperature with a magnetic stirring bar at $500 \mathrm{rpm}$ for a preset time period. Next, the particles were separated from the aqueous solution by filtration, making use of a polypropylene syringe filter with a pore size of $0.45 \mu \mathrm{m}$. The remaining metal ion concentration of the aqueous solution was measured with TXRF. The amount of metal ions adsorbed onto the chitosan-silica particles was then determined using the following formula:

$$
q_{e}=\frac{\left(c_{i}-c_{e}\right) V}{m_{a d s}}
$$

In this formula, $q_{e}$ is the amount of adsorbed metal ions at equilibrium ( $\mathrm{mmol} \mathrm{g}^{-1}$ adsorbent), $c_{i}$ is the initial metal ion concentration in aqueous solution $\left(\mathrm{mmol} \mathrm{L}^{-1}\right), c_{e}$ is the equilibrium metal ion concentration in aqueous solution $\left(\mathrm{mmol} \mathrm{L}^{-1}\right), V$ is the volume of the solution $(0.010 \mathrm{~L})$ and $m_{a d s}$ is the mass of the adsorbent $(0.025 \mathrm{~g})$.

\section{Reusability/stripping}

For the stripping experiments, initial adsorption took place in plastic centrifuge tubes. This allowed convenient separation of the aqueous solution from the loaded particles after $4 \mathrm{~h}$ of shaking, which was done by centrifugation (4200 rpm, $5 \mathrm{~min}$ ). The aqueous solution was analyzed with TXRF to determine the amount of $\mathrm{Nd}^{3+}$ ions that was adsorbed onto the sorbent. Then the particles were washed with MilliQ ${ }^{\circledR}$ water to remove non-complexated metal ions in the precipitate. Subsequently, the particles were stripped with $5 \mathrm{~mL}$ of a $1.0 \mathrm{M} \mathrm{HCl}$ solution with increasing concentration. The particles were vigorously shaken in the acid solution for $5 \mathrm{~min}$. 
The stripping solution was again removed by centrifugation at $4200 \mathrm{rpm}$ for $5 \mathrm{~min}$ and further analyzed to determine the amount of stripped ions. TXRF analysis required the use of polypropylene disks (instead of glass carriers), in order to be able to investigate the potential leaching of silicon.

The reusability experiments occurred similarly. After removing the aqueous solution by centrifugation, stripping of the loaded particles with $10 \mathrm{~mL}$ of $1.0 \mathrm{M} \mathrm{HCl}$ for $10 \mathrm{~min}$ and washing the particles with $5 \mathrm{~mL}$ of MilliQ ${ }^{\circledR}$ water, the particles were kept overnight at $40^{\circ} \mathrm{C}$. In a next adsorption cycle, $10 \mathrm{~mL}$ of aqueous $\mathrm{Nd}^{3+}$ solution was again added to the functionalized hybrid materials and shaken for $4 \mathrm{~h}$. The adsorption that resulted from the first, the second and the third reusability cycle were compared to the initial adsorption amount to determine the extraction efficiency in consecutive experiments.

\section{Separation experiments}

The setup was composed of a Büchi chromatography pump B-688, to control the pressure and the eluent flow, and a glass Büchi BOROSILIKAT 3.3 column tube, $\mathrm{N}^{\circ} 17988$ with dimensions $9.6 \mathrm{~mm} \times 115 \mathrm{~mm}$ (bed volume $=8.3 \mathrm{~mL})$. Separated compounds were collected with the aid of a Büchi Automatic Fraction Collector B-684. The distinct fractions were monitored by ex-situ analysis of the fractions with TXRF to determine the respective metal-ion concentrations.

The method of slurry-packing was used to pack the columns. Therefore, DTPA-chitosansilica $(2.0 \mathrm{~g})$ was soaked in an aqueous $\mathrm{HNO}_{3}$ solution, set at $\mathrm{pH} 3$, before pouring the stationary phase in the column. Pressurized air was used to ultimately pack the wet resin slurry. Metal frits were mounted at the top and at the bottom of the column, serving as filters that allow the mobile phase to pass, but keep the stationary phase inside the MPLC-column. Flow rates up to 20 $\mathrm{mL} / \mathrm{min}$ could be reached without exceeding the pressure maximum, set at 10 bar. The 
experiment was preceded by a thorough washing of the column with $50 \mathrm{~mL}$ of demineralized water, followed by conditioning of the column with $50 \mathrm{~mL}$ of an aqueous $\mathrm{HNO}_{3}$ solution, set at $\mathrm{pH}$ 1.50. Also the $\mathrm{Nd}^{3+} / \mathrm{Dy}^{3+}$ separation mixture was set at $\mathrm{pH} 1.50$ with $\mathrm{HNO}_{3}$. The feed concentration was $2.0 \mathrm{mM}$ for both metal ions, hence $\mathrm{Nd}^{3+}$ and $\mathrm{Dy}^{3+}$ were present in a 1:1 molar ratio. An average flow rate of $5 \mathrm{~mL} / \mathrm{min}$ was set. 


\section{RESULTS AND DISCUSSION}

\section{Synthesis}

Silica gels are most often synthesized by hydrolysis of monomeric, tetrafunctional alkoxide precursors employing a mineral acid or base as a catalyst. ${ }^{45}$ In the synthesis procedure described in this paper, tetraethyl orthosilicate (TEOS) was used as silica precursor and ammonia as a catalyst in a large excess of water. Because water and TEOS are immiscible, a mutual solvent such as ethanol is mostly used as a homogenizing agent. However, the gel could be prepared without addition of ethanol because the amount produced as by-product of the hydrolysis reactions was sufficient to homogenize the initially phase-separated system by strong stirring. ${ }^{45}$ In the subsequent condensation reactions, the formed silanol groups reacted to produce siloxane bonds. Base-catalyzed polymerization with large $\mathrm{H}_{2} \mathrm{O}$ :Si ratios produces highly condensed 'particulate' sols. ${ }^{45}$ As the chitosan amino groups remained available after hybridization, the chitosan-silica materials could easily be functionalized with EDTA and DTPA by grafting the corresponding anhydrides on the chitosan amino groups. The chemical structure of EDTAfunctionalized chitosan-silica is depicted in Figure 1. The structure of DTPA-chitosan-silica is similar.

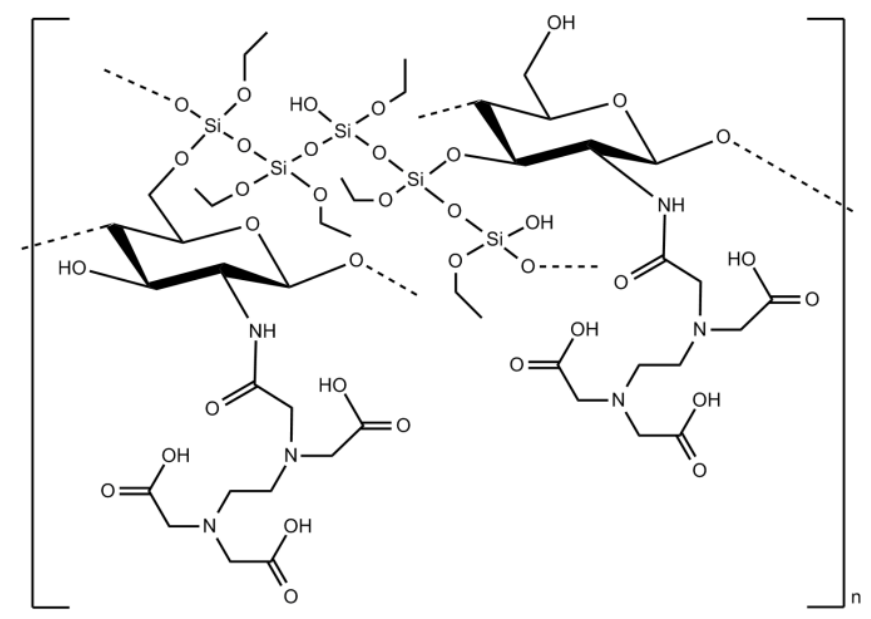

Figure 1: Chemical structure of EDTA-functionalized chitosan-silica. 


\section{Characterization}

The functional groups on the hybrid materials were investigated by FTIR. In all infrared spectra, a broad band was present between 3200 and $3600 \mathrm{~cm}^{-1}$, due to the symmetric vibration of free $\mathrm{NH}_{2}$ and $\mathrm{OH}$ groups. These originate mainly from chitosan, but also silanol groups from silica contribute to this band. Whereas in the spectra of the chitosan-silica materials only one peak around $1640( \pm 2) \mathrm{cm}^{-1}$ was present, several peaks occurred between 1350 and $1750 \mathrm{~cm}^{-1}$ in the spectra of the functionalized materials. These peaks arise from the presence of the carboxylic acid groups on the surface of the sorbents. The peak around $1640 \mathrm{~cm}^{-1}$ in the pre-functionalized chitosan-silica materials is due to the presence of an acetyl carbonyl group on part of the chitosan moieties. The most intense band in the spectra was found between 1090 and $1030 \mathrm{~cm}^{-1}$ and can be associated to the Si-O-Si and Si-O-C vibrations. This band confirms that the hybridization went well, together with the peak at $956( \pm 1) \mathrm{cm}^{-1}$, which occurs because of the $\mathrm{Si}-\mathrm{OH}$ stretch that is shifted from $950 \mathrm{~cm}^{-1}$ by hydrogen-bonding interactions.

The surface morphology was investigated by SEM (Figure 2). The surface changes upon hybridization are clear by comparison of Figure $2 \mathrm{a}$ and $2 \mathrm{~b}$. The encapsulation of chitosan flakes in a silica network creates a coarser surface. Nevertheless, the chitosan amine groups stay available for functionalization. This can be observed in Figures $2 \mathrm{c}$ and $2 \mathrm{~d}$, where it can also be seen that the SEM pictures resulting from EDTA- and DTPA-chitosan-silica are very similar. Functionalization seemingly leads to a more dense appearance of the polymer structures, which can be attributed to the fact that cross-linking occurs to some extent by functionalization with EDTA- and DTPA-bisanhydride. 


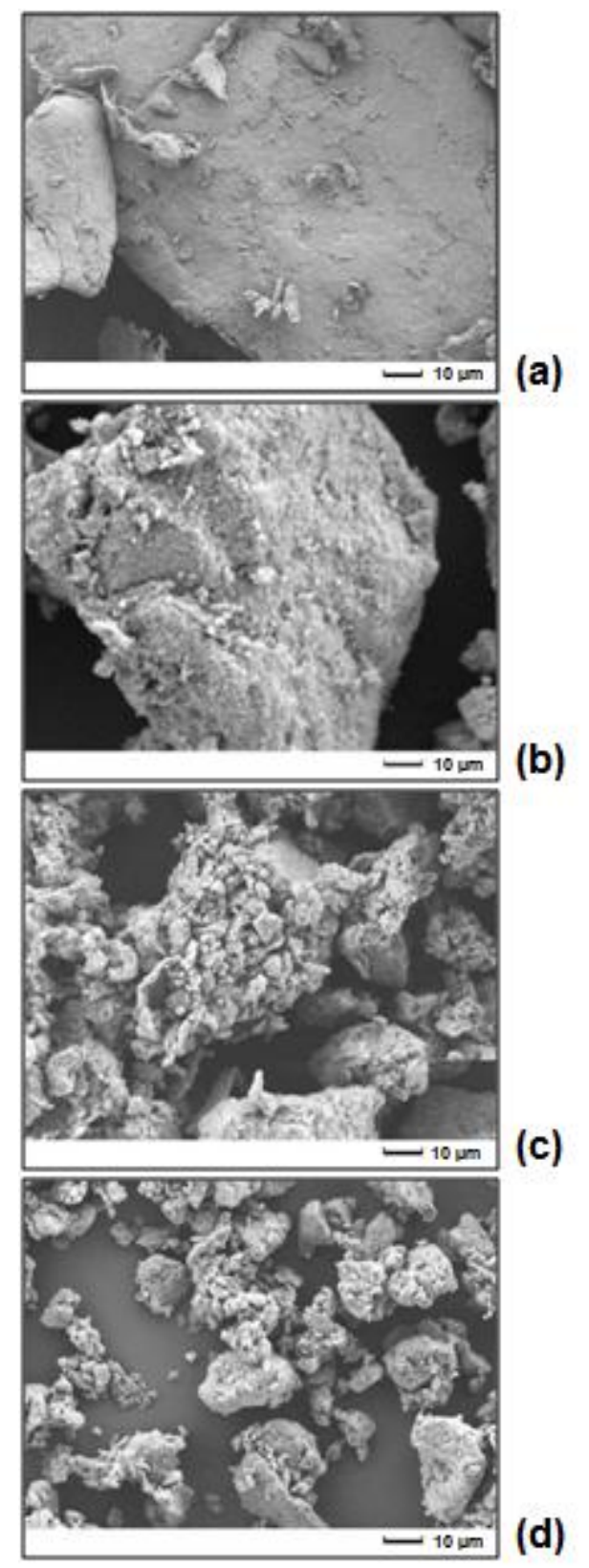

Figure 2: SEM images made at acceleration voltage: $5.0 \mathrm{kV}$; working distance: $15.2 \mathrm{~mm}$; photo magnification 1000x; (a) chitosan base material; (b) chitosan-silica; (c) EDTA-chitosan-silica; ( d) DTPA-chitosan-silica.

BET analysis was used to measure the specific surface and porosity of the material (Table 1). This technique is based on the multilayer adsorption of nitrogen as a function of relative pressure. The obtained data is very reproducible for both batches and thus the hybridization procedure can be considered to be highly reproducible. They clearly show the effect of hybridization. Firstly, it was seen that the specific surface area of hybridized chitosan is 
dramatically increased, which was also concluded from the SEM images. Secondly and more importantly, the porosity was increased remarkably because of the hybridization. As the pore size is between 2 and $50 \mathrm{~nm}$, these materials can be classified as mesoporous materials. This allows the use of these hybridized sorbents as chromatographic supports. It was experienced before that pure chitosan was not suited as a packing material for chromatographic separations as a consequence of column clogging because of its non-porous, elastic character. Only when mixed with sufficient amounts of silica, being porous and rigid, could a smooth flow be guaranteed over the entire column length. Hybridization increases the ease of use and the potential of this application.

Table 1: BET analysis results.

\begin{tabular}{cccc}
\hline & $\begin{array}{c}\text { Specific surface area } \\
\left(\mathrm{m}^{2} / \mathrm{g}\right)\end{array}$ & $\begin{array}{c}\text { Total pore volume } \\
\left(\mathrm{cm}^{3} / \mathrm{g}\right)\end{array}$ & $\begin{array}{c}\text { Average pore radius } \\
(\AA)\end{array}$ \\
\hline Chitosan & 1 & 0.003 & 40 \\
\hline Chitosan-silica $^{\mathrm{i}}$ & 218 & 1.024 & 94 \\
EDTA-chitosan-silica & 230 & 0.718 & 63 \\
\hline \multicolumn{2}{c}{ Chitosan-silica } \\
DTPA-chitosan-silica & 219 & 1.036 & 58 \\
\hline i & 198 & 0.573 & \\
ii $\quad$ A first batch of chitosan-silica was made to subsequently functionalize with EDTA.
\end{tabular}

Functionalization of the hybrid particles has a minor influence on the surface characteristics. The specific surface area fluctuates around the value of $215( \pm 15) \mathrm{m}^{2} / \mathrm{g}$. The porosity on the other hand decreases. It is not clear whether the presence of organic ligands causes filling of the (larger) pores or not. A cross-linking effect by the aminopolycarboxylic acid ligands would however explain why the decrease of total pore volume and average pore radius is higher for DTPA-chitosan-silica than for EDTA-chitosan-silica.

TGA measured the thermal stability of the particles. The shapes of the TGA curves are very similar for the non-functionalized and the functionalized hybrid particles. However, the 
functionalized chitosan-silica materials started to decompose at a lower temperature $\left(190{ }^{\circ} \mathrm{C}\right)$. The combustion products were analyzed by a coupled mass spectrometer. At $190{ }^{\circ} \mathrm{C}$, the carboxylic acid functions were released rapidly from the ligands. Further decomposition of the organic matrix occurred in all materials from $220{ }^{\circ} \mathrm{C}$ to $300{ }^{\circ} \mathrm{C}$ in a first stage. In this stage, small fragments evaporated and chitosan started to de-polymerize. The monomeric units on their turn decomposed until a constant weight was reached at $600{ }^{\circ} \mathrm{C}$. The main combustion products were $\mathrm{CO}_{2}, \mathrm{H}_{2} \mathrm{O}$ and $\mathrm{N}_{2}$, while $\mathrm{NH}_{3}$ or $\mathrm{NO}_{\mathrm{x}}$ compounds were not detected.

The ratio chitosan/silica in the respective materials was estimated by comparison of three independent techniques (TGA, ICP and CHN). The residual masses derived from the TGA measurements arise from the inorganic part and the loss-on-ignition (LOI) value is a measure for the organic content. ICP and CHN allowed determination of the amount of silicon and carbon, hydrogen and nitrogen, respectively. All values can be found in the ESI (TGA results in Table S1, ICP results in Table S2 and CHN results in Table S3). To calculate the organic content from the $\mathrm{CHN}$ measurements, all chitosan moieties were considered to be functionalized in EDTAand DTPA-chitosan-silica. This is realistic in case of EDTA-chitosan-silica, but less for DTPAchitosan-silica, as described in our earlier work. ${ }^{44}$

An overall average value was determined to get the percentages of chitosan and silica in the chitosan-silica particles (Table 2). Calculations show that the hybridization procedure is quite reproducible with an average ratio of 1 part of chitosan $(25 \mathrm{wt} \%)$ to 3 parts of silica $(75 \mathrm{wt} \%)$. By functionalization of the particles, the organic share obviously increased a little bit due to immobilization of organic ligands. Notice that this increase is higher for functionalization with EDTA than with DTPA. The apparent lower degree of functionalization for DTPA-chitosansilica can be attributed to a cross-linking effect. Nevertheless, the resulting ratios are still roughly comparable for EDTA-chitosan-silica and DTPA-chitosan-silica, both containing more specifically 3 parts of chitosan (30 wt $\%$ ) to 7 parts of silica (70 wt $\%)$. Finally, it was confirmed 
that a smooth flow could be obtained with these stationary phases by packing EDTA-chitosansilica and DTPA-chitosan-silica in a column. Therefore, it is possible to use these particles as a resin for the separation of rare earths by means of ion-exchange column chromatography.

Table 2: Average values for the chitosan and silica content in the (functionalized) chitosan-silica materials as a result of TGA, ICP and CHN measurements.

\begin{tabular}{|c|c|c|c|}
\hline & $\begin{array}{c}\text { Average organic } \\
\text { content }(\mathrm{wt} \%)\end{array}$ & $\begin{array}{l}\text { Average silica } \\
\text { content }(\mathrm{wt} \%)\end{array}$ & Ratio chitosan:silica \\
\hline Chitosan-silica $^{\mathrm{i}}$ & 25.8 & 74.2 & $1: 3$ \\
\hline EDTA-chitosan-silica & 31.0 & 69.0 & $3: 7$ \\
\hline Chitosan-silica $^{\mathrm{ii}}$ & 25.8 & 74.2 & $1: 3$ \\
\hline DTPA-chitosan-silica & 29.7 & 70.3 & $3: 7$ \\
\hline
\end{tabular}

A first batch of chitosan-silica was made to subsequently functionalize with EDTA.

ii A second batch chitosan-silica was made to subsequently functionalize with DTPA.

\section{Luminescence}

In order to investigate the structure of the complexes of rare-earth ions with the EDTA or DTPA groups on chitosan-silica and in order to determine the number of coordinated water molecules in the first coordination sphere, the luminescence properties of $\mathrm{Eu}^{3+}$-loaded chitosan-silica were measured. The excitation spectrum was dominated by a peak at $394.90 \mathrm{~nm}$ (corresponding to the ${ }^{7} \mathrm{~F}_{0} \rightarrow{ }^{5} \mathrm{~L}_{6}$ transition), so the emission spectrum was recorded by irradiation of the sample with this wavelength (Figure 3). 


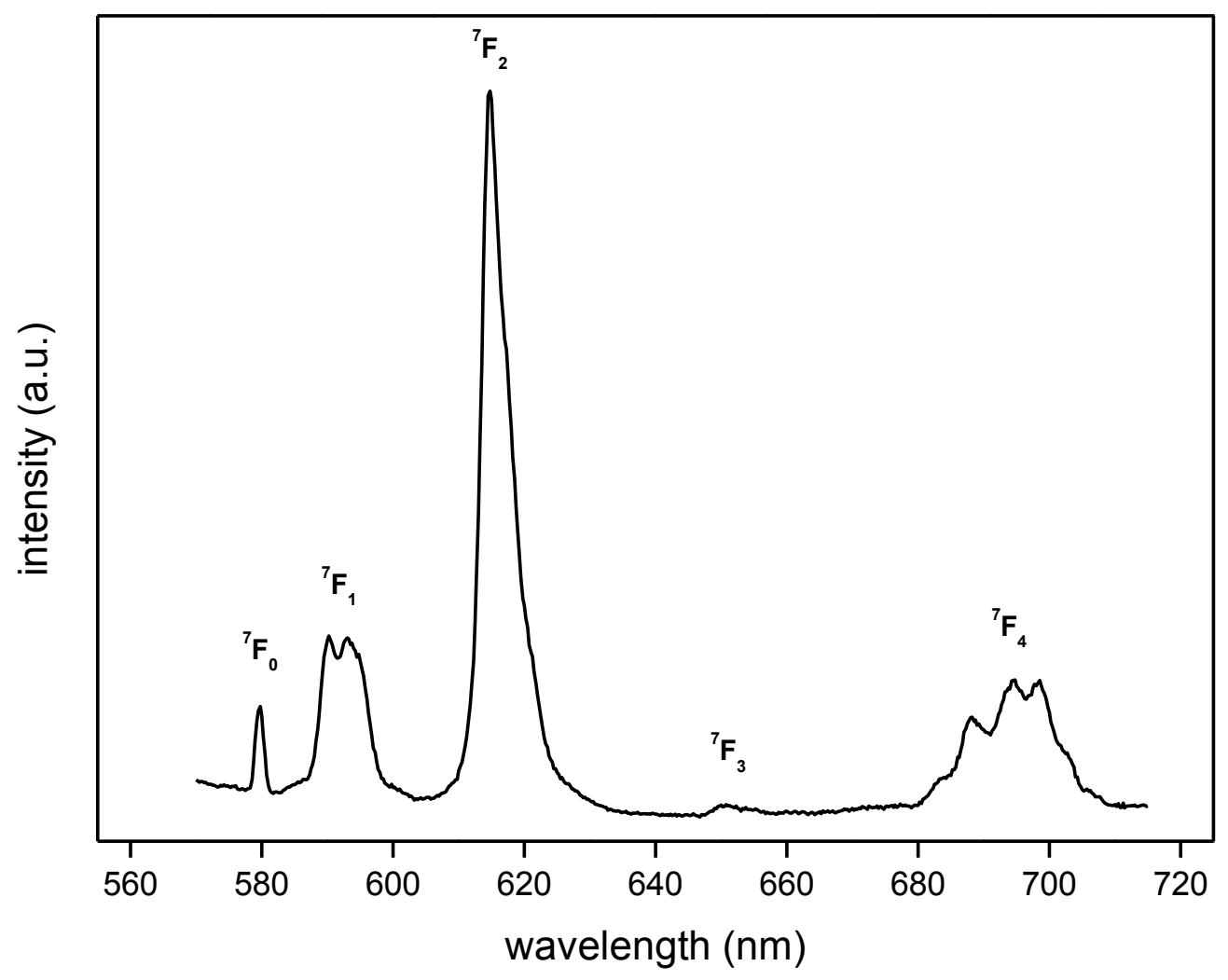

Figure 3: Emission spectrum of Eu(III)-coordinated EDTA-chitosan-silica $\left(\lambda_{e x c}=394.90 \mathrm{~nm}\right.$, room temperature).

The transitions in the spectrum all originate from the ${ }^{5} \mathrm{D}_{0}$ level and terminate at various ${ }^{7} \mathrm{~F}_{J}$ levels $(J=0-4$, indicated in the figure). The pattern, shape and relative intensities of the peaks provide information about the environment of the $\mathrm{Eu}^{3+}$ ion. Since the ${ }^{5} \mathrm{D}_{0} \rightarrow{ }^{7} \mathrm{~F}_{2}$ hypersensitive transition is the most intense transition in the $\mathrm{Eu}^{3+}$ ion coordinated EDTA-chitosan-silica material (and more intense than the ${ }^{5} \mathrm{D}_{0} \rightarrow{ }^{7} \mathrm{~F}_{1}$ transition), this indicates that no centrosymmetry is present. The presence of the ${ }^{5} \mathrm{D}_{0} \rightarrow{ }^{7} \mathrm{~F}_{0}$ transition indicates that the point-group symmetry of the Eu(III)-site is $\mathrm{C}_{\mathrm{n}}, \mathrm{C}_{\mathrm{nv}}$ or $\mathrm{C}_{\mathrm{s}}{ }^{46}$ The fact that this transition appears as a single peak indicates that $\mathrm{Eu}^{3+}$ ions occupy no more than one site of symmetry.

The hydration number $q$ of the $\mathrm{Eu}^{3+}$ ion coordinated to functionalized chitosan-silica was determined. This was done by recording the decay time of the ${ }^{5} \mathrm{D}_{0}$ excited state (measured by 
monitoring the luminescence intensity of the ${ }^{5} \mathrm{D}_{0} \rightarrow{ }^{7} \mathrm{~F}_{2}$ hypersensitive transition at $613.50 \mathrm{~nm}$ ) for the $\mathrm{Eu}(\mathrm{III})$-coordinated functionalized chitosan-silica suspended in $\mathrm{H}_{2} \mathrm{O}$ and $\mathrm{D}_{2} \mathrm{O}$ and by applying a simplified form of the modified Horrocks-Supkowski formula: ${ }^{47}$

$$
q=1.11 \times\left(\frac{1}{\tau_{\mathrm{H}_{2} \mathrm{O}}}-\frac{1}{\tau_{D_{2} \mathrm{O}}}-0.31\right)
$$

$\tau_{\mathrm{H}_{2} \mathrm{O}}$ and $\tau_{\mathrm{D}_{2} \mathrm{O}}$ are the luminescence decay times determined in water and deuterated water, respectively (Table 4). The rounded hydration number was 3 for EDTA-chitosan-silica and 1 for DTPA-chitosan-silica. Assuming that $\mathrm{Eu}^{3+}$ coordinates with five atoms of the EDTA-moiety (two nitrogen atoms and three oxygen atoms) and seven atoms of the DTPA-moiety (three nitrogen atoms and four oxygen atoms), this means a coordination number of eight for the adsorbed $\mathrm{Eu}^{3+}$-ion in both materials. In that view a bicapped trigonal prismatic polyhedron with $\mathrm{C}_{\mathrm{s}}$ symmetry could possibly be the symmetry of the coordinated complexes.

Table 3: Lifetimes of the ${ }^{5} D_{0}$ excited state for Eu(III)-coordinated EDTA-and DTPA-chitosan-silica in water and deuterated water and corresponding hydration numbers by application of Eq. (2).

\begin{tabular}{lccc}
\hline & $\boldsymbol{\tau}_{\mathbf{H}_{\mathbf{2}} \mathbf{0}}(\mathrm{ms})$ & $\boldsymbol{\tau}_{\mathbf{D}_{\mathbf{2}} \mathbf{0}}(\mathrm{ms})$ & $\mathbf{q}$ \\
\hline EDTA-chitosan-silica & 0.286 & 1.738 & 2.89 \\
DTPA-chitosan-silica & 0.534 & 1.629 & 1.05 \\
\hline
\end{tabular}

\section{Kinetics of adsorption}

The influence of contact time, $\mathrm{pH}$ and adsorbent mass were investigated for aqueous nitrate solutions of $\mathrm{Nd}^{3+}$ as a model system for all rare-earth ions. For each experiment stirring occurred at room temperature with a magnetic stirring bar at $500 \mathrm{rpm}$. The influence of contact time on adsorption of rare-earth ions by EDTA- and DTPA-functionalized chitosan-silica is shown in Figure 4. The aqueous feed had an initial concentration of $0.50( \pm 0.01) \mathrm{mmol} \mathrm{L}^{-1}$ in this 
experiment. The $\mathrm{pH}$ was not adjusted. The initial $\mathrm{pH}$ of 6.0 evolved to an equilibrium $\mathrm{pH}$ of 3.0 during the experiment due to the release of carboxylic acid protons when binding rare-earth ions.

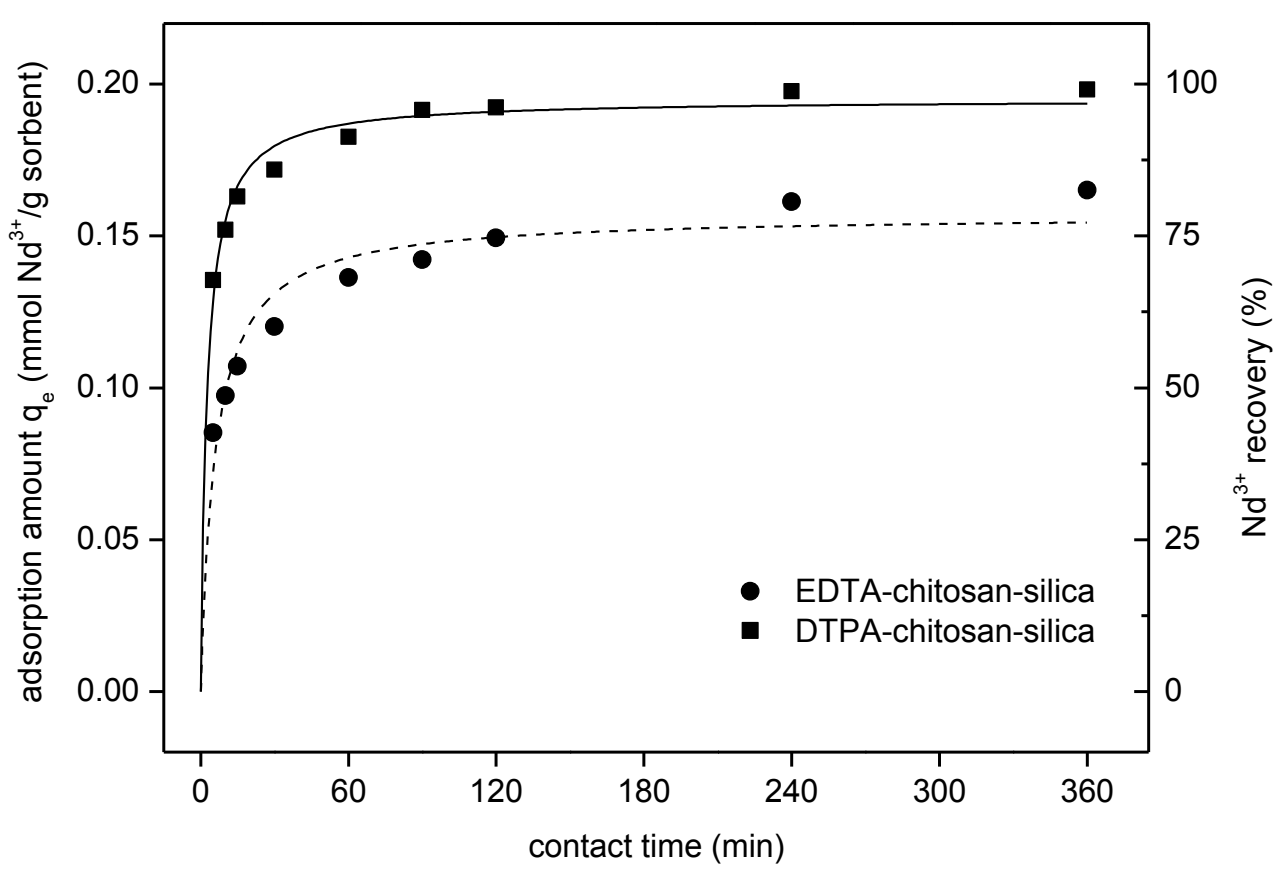

Figure 4: Kinetics of adsorption of $\mathrm{Nd}^{3+}$ by EDTA-chitosan-silica and DTPA-chitosan-silica. Data points were fitted with the pseudo-second-order kinetic model.

EDTA- and DTPA-chitosan-silica show a similar kinetic profile due to their similar structure.

The major part of the present metal ions is already coordinated to the adsorbents within one hour. DTPA-chitosan-silica shows a higher adsorption amount than EDTA-chitosan-silica. In these experimental conditions, $25 \mathrm{mg}$ of DTPA-chitosan-silica proved sufficient to adsorb all $\mathrm{Nd}^{3+}$ ions from $10 \mathrm{~mL}$ solution $\left(c_{a q}=0.50 \mathrm{mmol} \mathrm{L}^{-1}\right)$, while $25 \mathrm{mg}$ of EDTA-chitosan-silica adsorbed only $80 \%$ of the ions present. Data points were fitted with the pseudo-second-order kinetic model in order to predict the rate of adsorption (Table 4). The pseudo-second-order model is given by the following equation:

$$
q_{t}=\frac{q_{e}^{2} k t}{1+q_{e} k t}
$$


where $q_{t}$ and $q_{e}(\mathrm{mmol} / \mathrm{g})$ the amount of metal ions adsorbed at time $t$ and at equilibrium, respectively, and $k$ the pseudo-second-order rate constant. In the pseudo-second-order model, the rate-limiting step is the surface reaction. As the $\mathrm{R}^{2}$ value is higher than 0.90 for both materials, the chemisorption can be assumed to be the rate limiting step, rather than the pore diffusion. Data fitting with the intraparticle diffusion model (in analogy with work by Repo et al. ${ }^{34}$ ) was not satisfying with our data and thus not discussed.

Table 4: Results of fitting kinetic data with the pseudo-second-order kinetic model.

\begin{tabular}{lccc}
\hline & $\begin{array}{c}\mathbf{q}_{\mathbf{e}} \\
(\mathrm{mmol} / \mathrm{g})\end{array}$ & $\begin{array}{c}\mathbf{k} \\
(\mathrm{g} \mathrm{mmol} / \mathrm{min})\end{array}$ & $\mathbf{R}^{\mathbf{2}}$ \\
\hline EDTA-chitosan-silica & 0.16 & 1.07 & 0.91 \\
DTPA-chitosan-silica & 0.20 & 1.99 & 0.95 \\
\hline
\end{tabular}

The plateau value was reached after $3 \mathrm{~h}$ for EDTA-chitosan-silica and already after $2 \mathrm{~h}$ for DTPA-chitosan-silica as by then no more $\mathrm{Nd}^{3+}$ ions were in solution. All following adsorption experiments were performed for $4 \mathrm{~h}$ in order to ensure equilibrium conditions.

\section{Influence of aqueous pH}

The $\mathrm{pH}$ is one of the main parameters having an influence on the adsorption of metal ions, due to the protonation of complexing carboxylic acid groups on the surface of the sorbents. For both EDTA- and DTPA-chitosan-silica, the $\mathrm{pH}$ of the aqueous feed was varied between 1.0 and 7.0 (Figure 5). Because of hydrolysis of rare-earth ions, it does not make sense to investigate alkaline $\mathrm{pH}$ values. From the solubility constant of $\mathrm{Nd}(\mathrm{OH})_{3}, K_{s p}=10^{-23}$, it can be calculated that precipitation occurs from a $\mathrm{pH}$ of 7.37 in the concentration conditions of this experiment $\left(c_{a q}=\right.$ $\left.0.51 \mathrm{mmol} \mathrm{L}^{-1}\right){ }^{48}$ 


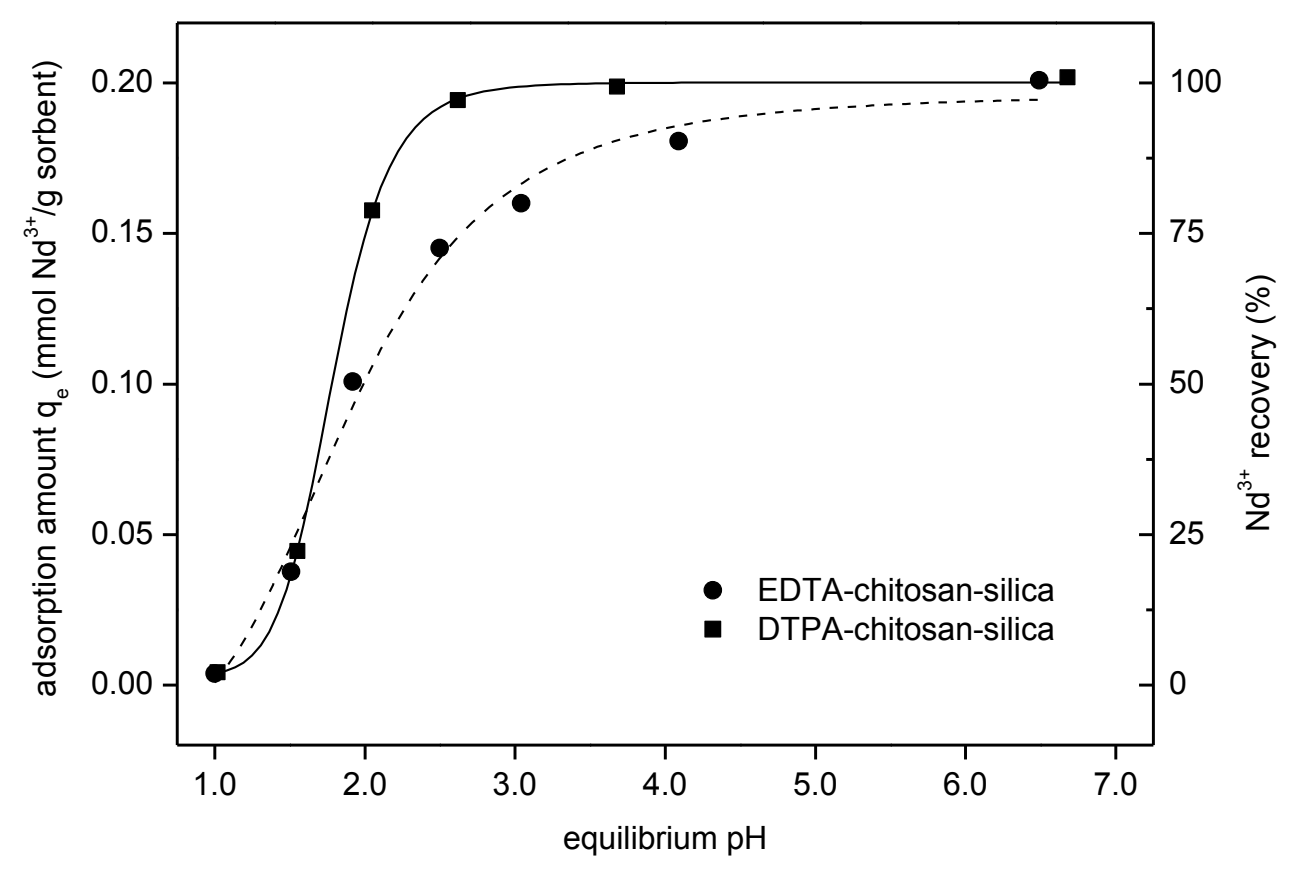

Figure 5: Influence of aqueous $\mathrm{pH}$ on the adsorption of $\mathrm{Nd}^{3+}$ by EDTA-chitosan-silica and DTPAchitosan-silica.

Adsorption increases in a sigmoidal way for both EDTA-chitosan-silica and DTPA-chitosansilica. No adsorption occurs at $\mathrm{pH} 1.0$ since the functional groups are fully protonated at this $\mathrm{pH}$. Raising the $\mathrm{pH}$ leads to a fast increase in adsorption amount. Whereas the increase in adsorption of $\mathrm{Nd}^{3+}$ continues for EDTA-chitosan-silica until $\mathrm{pH} 7$, at which all $\mathrm{Nd}^{3+}$ ions are recovered from solution, the plateau value is already reached at $\mathrm{pH} 4$ for DTPA-chitosan-silica. The adsorption amount of $0.20 \mathrm{mmol} \mathrm{Nd} d^{3+} / \mathrm{g}$ sorbent corresponds to complete recovery of the $\mathrm{Nd}^{3+}$ ions present in solution, so that it can be expected that adsorption with DTPA-chitosan-silica would also proceed at higher $\mathrm{pH}$ with higher metal ion concentrations. Note also that the equilibrium $\mathrm{pH}$ is approximately 3 in a standard experiment in which no $\mathrm{pH}$ adjustments are made, which is a consequence of the exchange of sorbent protons for metal ions during the adsorption reaction. Hence, this explains the observed adsorption amounts in the previous experiment (Figure 4), 
being 0.16 and $0.20 \mathrm{mmol} \mathrm{Nd} \mathrm{m}^{3+} / \mathrm{g}$ sorbent for EDTA-chitosan-silica and DTPA-chitosan-silica, respectively.

\section{Adsorption isotherms}

As a third parameter, the adsorbent mass was varied. By increasing the adsorbent mass, a decrease of the $\mathrm{Nd}^{3+}$ equilibrium concentration occurs. By plotting the adsorption amount versus the equilibrium concentration, adsorption isotherms were obtained (Figure 6).

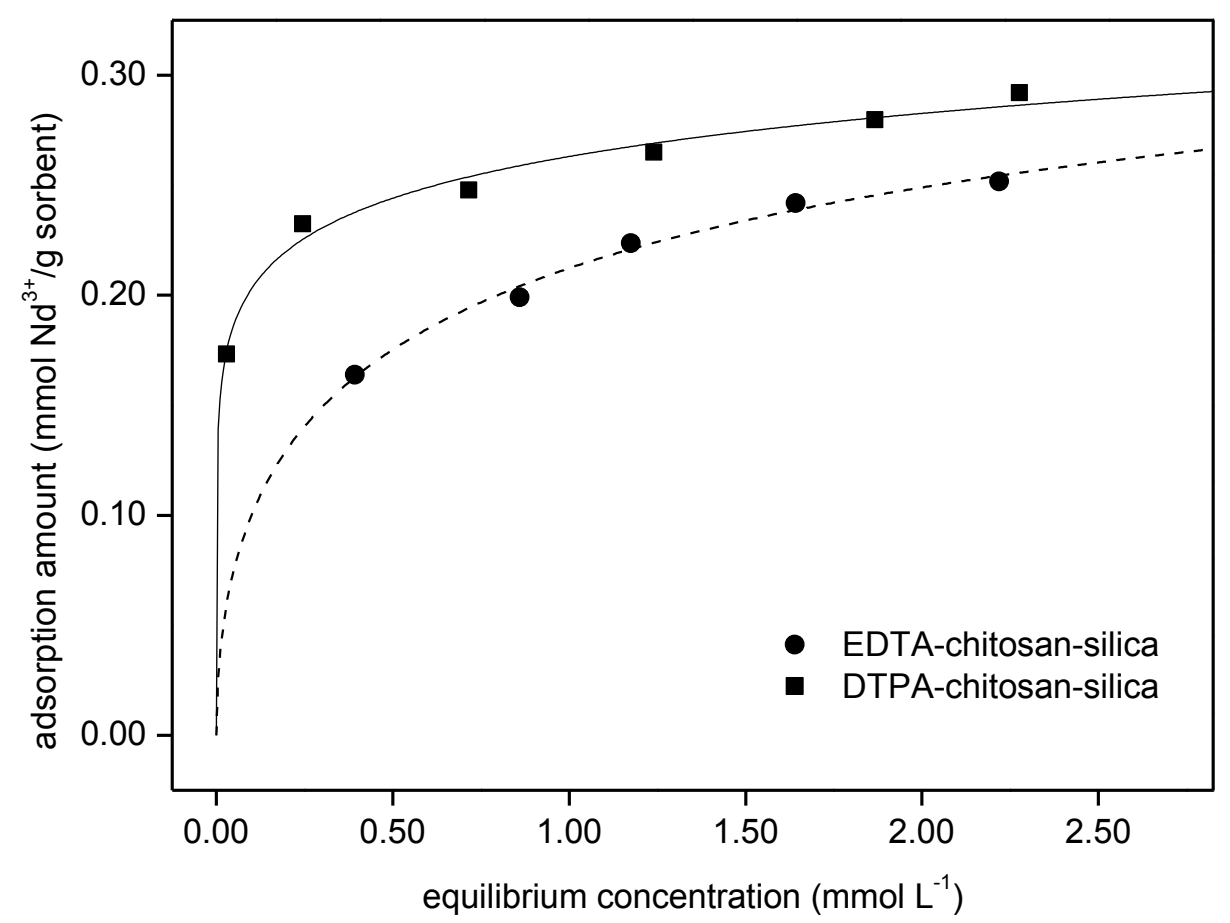

Figure 6: Adsorption isotherms of EDTA-chitosan-silica and DTPA-chitosan-silica for the adsorption of $\mathrm{Nd}^{3+}$, both fitted with the Langmuir-Freundlich model.

To characterize the sorption equilibria, data points were fitted with two commonly used sorption models: the Langmuir adsorption model and the Langmuir-Freundlich model. The Langmuir adsorption model (Eq. 4) is based on the fact that a solid surface has a finite amount of sorption sites. 


$$
q_{e q}=q_{\max }\left(\frac{K_{L} C_{e q}}{1+K_{L} C_{e q}}\right)
$$

The sorption process occurs in a monolayer that covers the surface of the material. It is further assumed that adsorption is a dynamical process. At equilibrium, the number of adsorbed ions equals the number of ions that are released from the adsorbent surface. ${ }^{49}$ Fitting results for the Langmuir method are shown in Table 5. The $\mathrm{R}^{2}$ value that results from the fitting procedure was low for DTPA-chitosan-silica, which indicated that the original Langmuir model was not appropriate to describe the adsorption of $\mathrm{Nd}^{3+}$ by this material.

Table 5: Fitting results of adsorption isotherm data with Langmuir model.

\begin{tabular}{lccc}
\hline & $\begin{array}{c}\text { theoretical } \mathbf{q}_{\max } \\
\left(\mathrm{mmol} \mathrm{Nd}^{3+} / \mathrm{g} \text { sorbent }\right)\end{array}$ & $\begin{array}{c}\mathbf{K}_{\mathbf{L}} \\
(\mathrm{L} / \mathrm{mmol})\end{array}$ & $\mathbf{R}^{\mathbf{2}}$ \\
\hline EDTA-chitosan-silica & 0.27 & 3.72 & 0.95 \\
DTPA-chitosan-silica & 0.27 & 55.13 & 0.85 \\
\hline
\end{tabular}

$\mathrm{K}_{\mathrm{L}}=$ Langmuir isotherm constant

The Langmuir-Freundlich model (Eq. 5) is a modified version of the original Langmuir model, based on the Freundlich equation, which is the earliest known relationship describing non-ideal and reversible adsorption, not restricted to the formation of a monolayer. This empirical model can be applied to multilayer adsorption, with non-uniform distribution of adsorption sites and affinities over the heterogeneous surface. ${ }^{50}$ The results for the Langmuir-Freundlich method are shown in Table 6. As it can be seen from the $\mathrm{R}^{2}$ value that this model is more accurate, it was chosen to fit the data in Figure 6 with the Langmuir-Freundlich model.

$$
q_{e q}=q_{\max }\left(\frac{\left(K_{L F} C_{e q}\right)^{n}}{1+\left(K_{L F} C_{e q}\right)^{n}}\right)
$$


Table 6: Fitting results of adsorption isotherm data with Langmuir-Freundlich model.

\begin{tabular}{lcccc}
\hline & $\begin{array}{c}\text { theoretical } \mathbf{q}_{\max } \\
\left(\mathrm{mmol} \mathrm{Nd}^{3+} / \mathrm{g}\right. \\
\text { sorbent })\end{array}$ & $\begin{array}{c}\mathbf{K}_{\mathbf{L F}} \\
(\mathrm{L} / \mathrm{mmol})\end{array}$ & $\mathbf{n}$ & $\mathbf{R}^{\mathbf{2}}$ \\
\hline EDTA-chitosan-silica & 0.42 & 1.09 & 0.52 & 0.99 \\
DTPA-chitosan-silica & 0.74 & 0.02 & 0.16 & 0.98 \\
\hline
\end{tabular}

$\mathrm{K}_{\mathrm{LF}}=$ Langmuir-Freundlich isotherm constant

Most important is the confirmation that DTPA-chitosan-silica has an overall better adsorption capacity than EDTA-chitosan-silica. The data also confirm the adsorption amounts obtained in upper experiments. These were performed with an aqueous $\mathrm{Nd}(\mathrm{III})$ concentration of $0.51 \mathrm{mM}$, for which adsorption amounts of $0.16 \mathrm{mmol} / \mathrm{g}$ for EDTA-chitosan-silica and $0.24 \mathrm{mmol} / \mathrm{g}$ for DTPA-chitosan-silica can be derived from Figure 6. Next, it can be concluded from the modeling that the maximum adsorption capacity of both materials (at high feed concentration) is higher than expected from the previously described experiments, up to $0.75 \mathrm{mmol} \mathrm{Nd}^{3+} / \mathrm{g}$ of DTPAchitosan-silica. This is in agreement with the observation that the Langmuir-Freundlich model fits the adsorption isotherms well for both materials. The Langmuir-Freundlich model supports the hypothesis that no simple monolayer of rare-earth ions is formed around the particles. The experimental validation of even higher adsorption amounts was not considered relevant, since the application of this type of materials is mainly the recovery of rare earths from diluted aqueous waste streams.

\section{Stripping and reusability studies}

After adsorption of rare-earth ions from solution, stripping of the immobilized ions is required for further processing and regeneration of the sorbent. This can be done by bringing the loaded 
adsorbents in contact with acidic solutions and shaking for $5 \mathrm{~min}$. The effect of the $\mathrm{HCl}$ concentration on the amount of stripping is shown for EDTA-chitosan-silica and DTPAchitosan-silica in Figure 7. The adsorbent materials were loaded by adsorption from an aqueous $\mathrm{Nd}^{3+}$ solution $\left(c_{a q}=1.05 \mathrm{mM}\right)$. The adsorption amount was equal to $0.22( \pm 0.01) \mathrm{mmol} / \mathrm{g}$ for EDTA-chitosan-silica and $0.25( \pm 0.02) \mathrm{mmol} / \mathrm{g}$ for DTPA-chitosan-silica. The experiment was performed in duplicate. Stripping in the less acidic region is easier for EDTA-chitosan-silica than for DTPA-chitosan-silica. This is in line with previous observations, in the sense that binding of $\mathrm{Nd}^{3+}$ ions is weaker for EDTA-chitosan-silica than for DTPA-chitosan-silica. The higher adsorption capacity of DTPA-chitosan-silica is reflected here in the observation that complex formation seems stronger so that higher concentrations of $\mathrm{HCl}$ are needed to desorb the $\mathrm{Nd}^{3+}$ ions from the particles.

Stripping solutions were also analyzed with TXRF to investigate the possible deterioration due to silicon leaching. No significant silicon leaching $(<1 \%$ particle loss $)$ was observed for all investigated $\mathrm{HCl}$ concentrations. It can be concluded that the material is stable within the time range needed for complete stripping of the loaded chitosan-silica. 


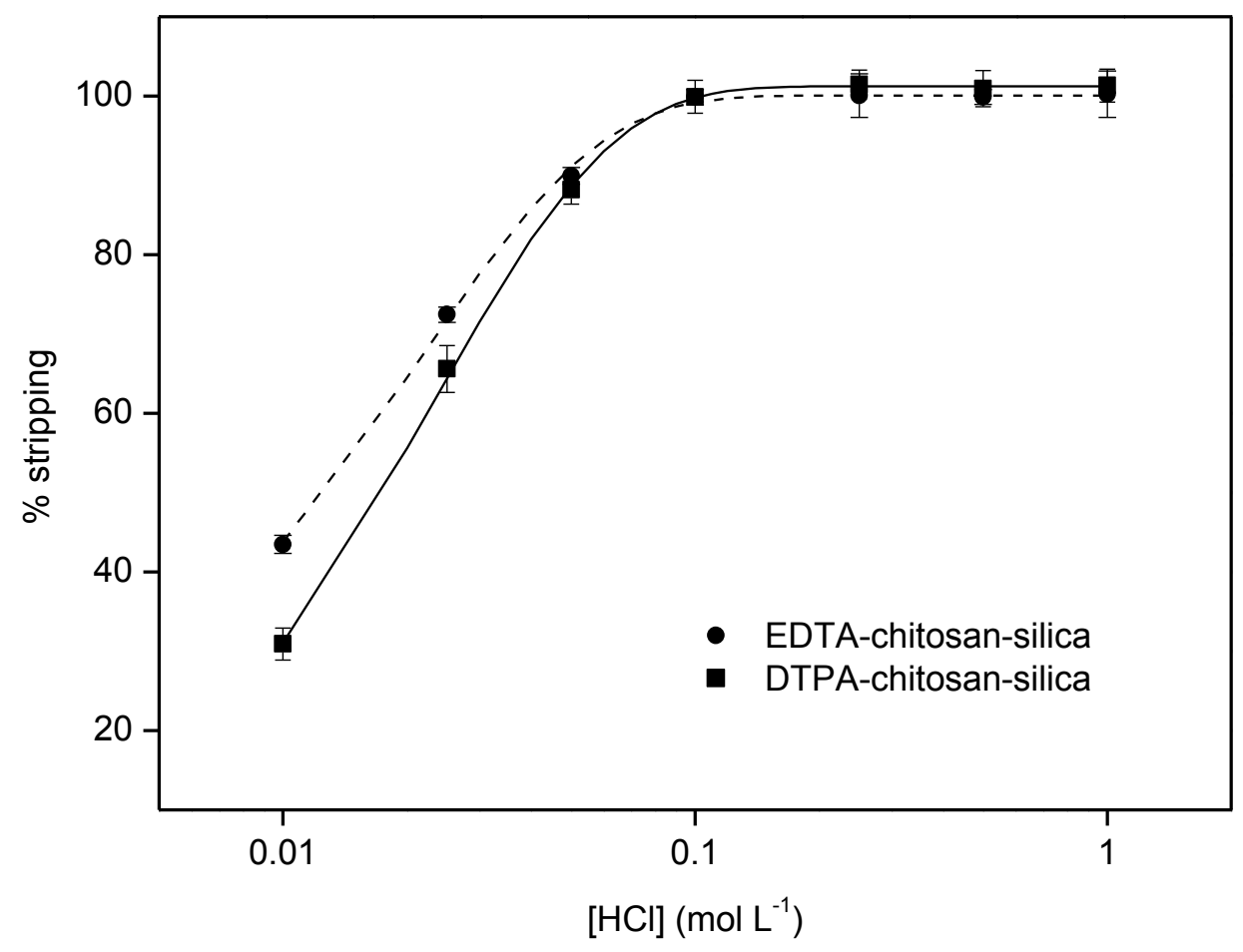

Figure 7: Effect of aqueous HCl concentration on the stripping efficiency for EDTA-chitosan-silica and DTPA-chitosan-silica.

The effect of stripping on the remaining adsorption performance was investigated for both materials. After loading the adsorbents with $\mathrm{Nd}^{3+}$ and stripping them for 5 min with a $1.0 \mathrm{M} \mathrm{HCl}$ aqueous solution, the particles were washed with demineralized water and reused in three consecutive adsorption/desorption cycles. The experiments were repeated in triplicate to reduce the experimental error. The results are shown in Figure 8. A drop in efficiency occurs for both materials after the first stripping cycle. In the following cycles, the adsorption amount remains quite constant, around 85\% for both EDTA-chitosan-silica and DTPA-chitosan-silica. The initial efficiency drop cannot be ascribed to inflicted damage of the silica network since it was described above that no silicon leaching was observed. Therefore, a more plausible explanation is that damages occur to the more fragile organic part of the adsorbents. It can be assumed that a small part of the functional groups is lost upon the first treatment with $1.0 \mathrm{M} \mathrm{HCl}$. Since the adsorption amount remains constant after the first cycle, it is assumed that the resulting material 
is strong enough to resist consecutive acidic stripping steps. As a consequence of these results, it can be claimed that both EDTA-chitosan-silica and DTPA-chitosan-silica are reusable, resulting in very sustainable materials.

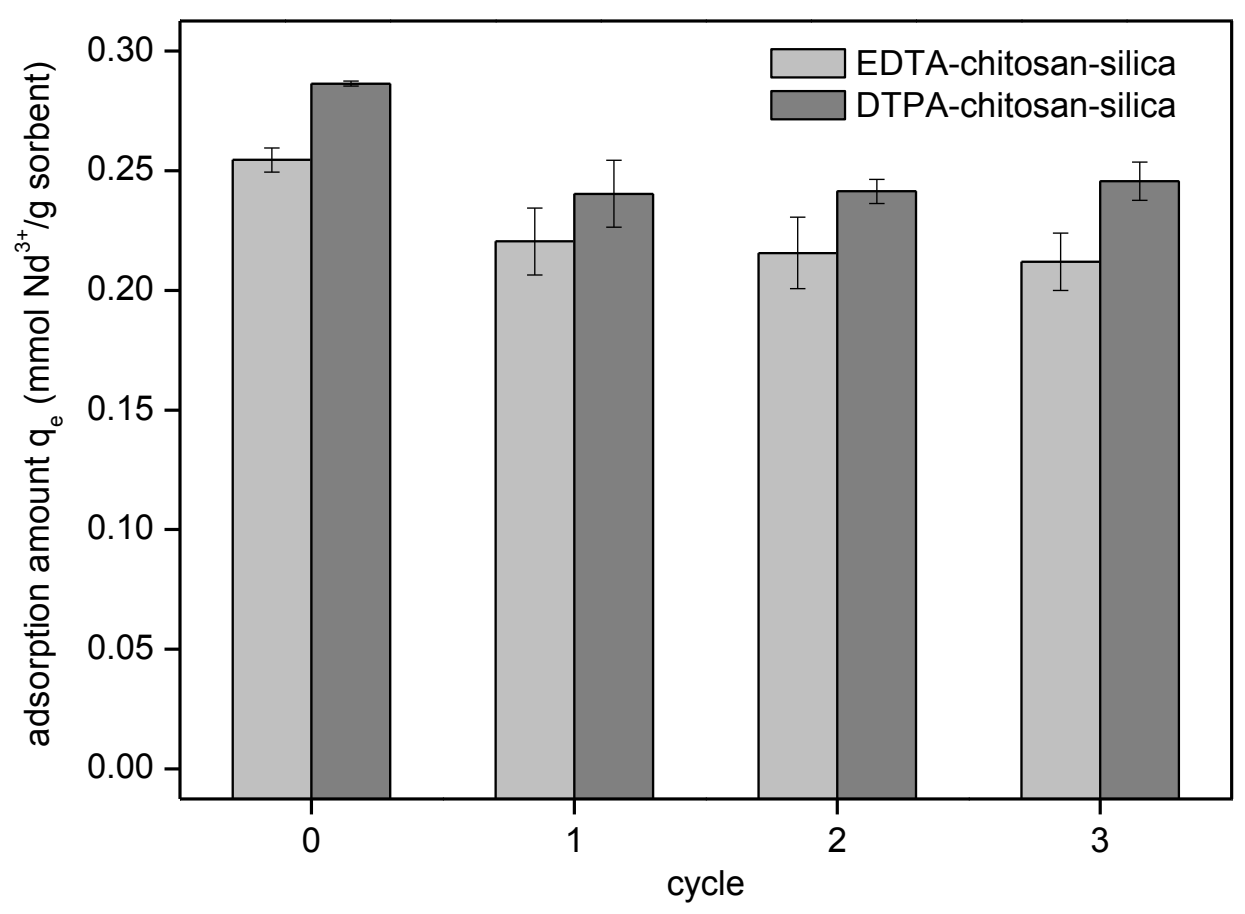

Figure 8: Adsorption amount for EDTA-chitosan-silica and DTPA-chitosan-silica in consecutive adsorption/desorption cycles.

\section{Investigation of selectivity}

Selectivity arises from differences in affinity of different metal ions for a selected material.

Therefore, it was first investigated whether differences exist in the adsorption amount of several rare-earth ions from different mono-component solutions. Batch adsorptions were performed with DTPA-chitosan-silica in aqueous solutions of $\mathrm{La}^{3+}, \mathrm{Nd}^{3+}, \mathrm{Eu}^{3+}, \mathrm{Dy}^{3+}$ and $\mathrm{Lu}^{3+}\left(c_{a q}=0.75\right.$ mmol L ${ }^{-1}$ ) as a function of equilibrium $\mathrm{pH}$. Distribution coefficients $D$ can then be calculated, which have been defined in the context of adsorption studies as:

$$
D=1000 \frac{m L}{L} \times \frac{q_{e}}{c_{e}}
$$


Here $q_{e}$ is the equilibrium adsorption amount $\left(\mathrm{mmol} \mathrm{g}^{-1}\right)$ and $c_{e}$ is the equilibrium concentration in solution $\left(\mathrm{mmol} \mathrm{L}^{-1}\right)$. Differences in affinity among the different lanthanide ions become clear from Figure 9 as the data points for the different ions are well distinct from each other.

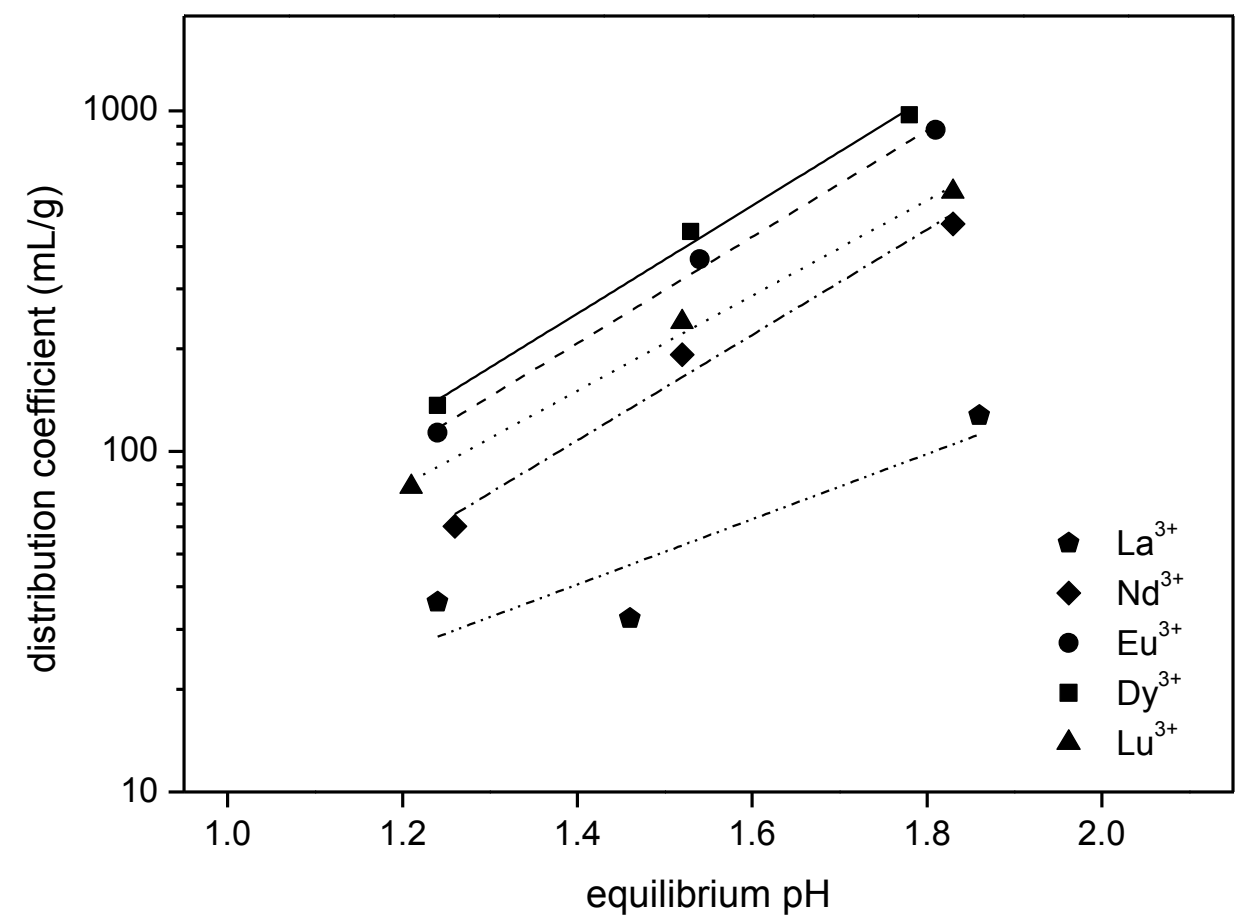

Figure 9: Distribution coefficients for adsorption of different lanthanide ions from mono-component solutions with DTPA-chitosan-silica as a function of equilibrium $p H$. Notice the logarithmic scale on the Y-axis.

The order of affinity among the metal ions perfectly follows the corresponding stability constants between the lanthanide ions and non-immobilized DTPA, as is depicted in Figure 1. The absolute values differ slightly as one carboxylic acid function less is available in immobilized DTPA, but the same trend is valid. The affinity for DTPA-chitosan-silica increases from lanthanum to dysprosium/holmium. This has to do with the lanthanide contraction, being the more than expected decrease in ion size for consecutive lanthanide ions. This is a consequence of the poor shielding of the nuclear charge by the $4 \mathrm{f}$ subshell, which causes the $5 \mathrm{~s}$ and $5 \mathrm{p}$ electrons to 
experience a larger effective nuclear charge. The smaller the ionic radius, the better the arms of DTPA can enfold the respective ion, resulting in stronger coordination. However, this phenomenon is characterized by an optimum size, as can be concluded from decreasing stability constants from erbium on. The smaller size of lutetium does not allow a strong coordination with the four arms of the large DTPA-ligand, explaining the position of its affinity curve between that of neodymium and europium (Figure 9).

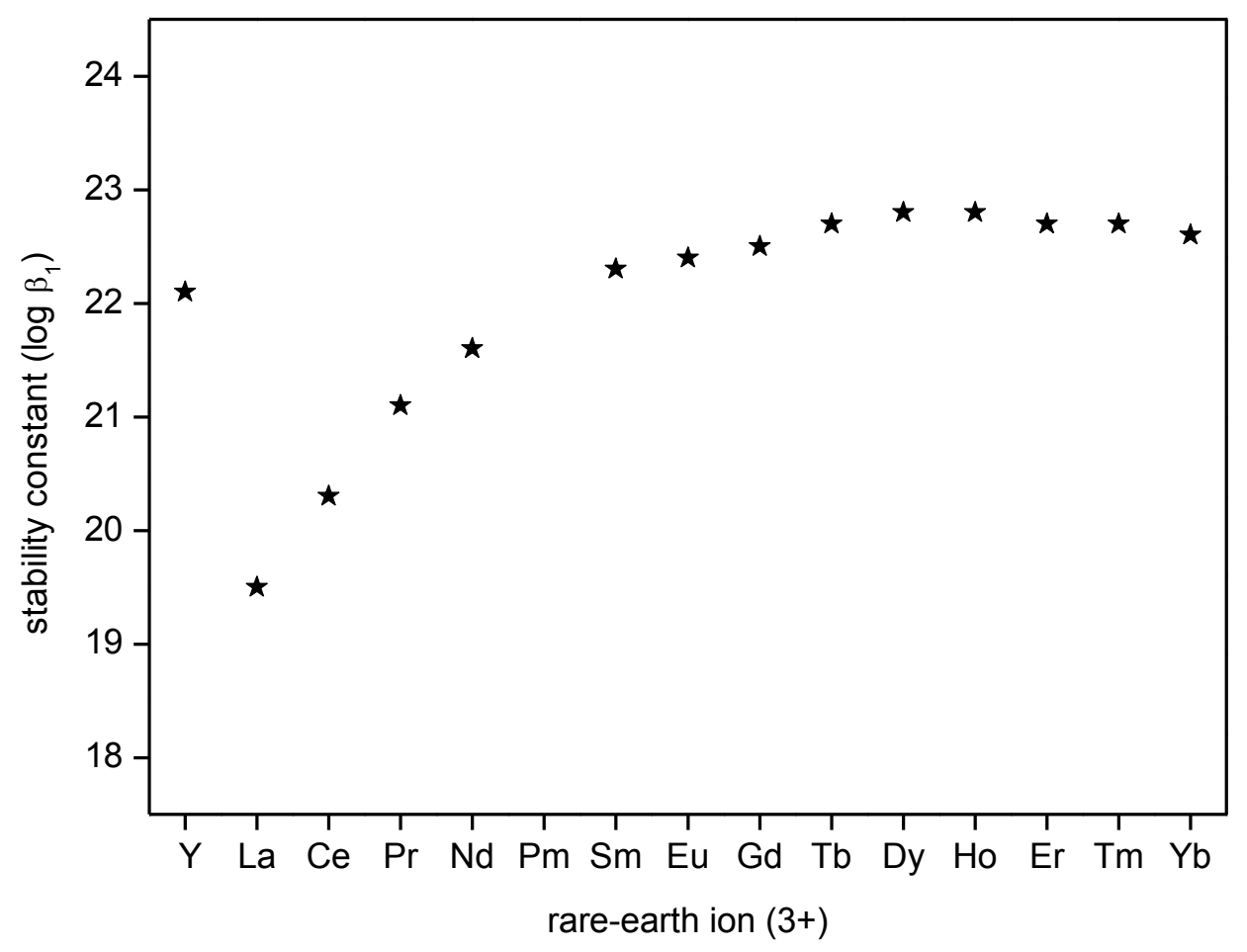

Figure 10: Literature stability constants $\left(\log \beta_{1}\right)$ for the trivalent rare-earth ions with nonimmobilized DTPA. ${ }^{51}$

Differences in affinity can be exploited to gain selectivity in multi-component solutions. The most important difference with adsorption experiments in mono-component solutions is that effective competition occurs between the different metal ions present in a mixture. Because the number of adsorption sites is limited, it is expected that the number of adsorption sites occupied 
by the different metal ions will depend on the affinity of that specific metal ion for the adsorbent. To confirm this statement, the same five lanthanides ( $\mathrm{La}, \mathrm{Nd}, \mathrm{Eu}, \mathrm{Dy}$ and $\mathrm{Lu}$ ) were used in a mixture $\left(c_{\mathrm{Ln}}^{3+}=0.44 \mathrm{mmol} \mathrm{L}{ }^{-1}\right)$ and subjected to adsorption by DTPA-chitosan-silica. The results of this experiment can be found in Figure 11. It becomes clear from this experiment that, the same trends as in mono-component solutions are valid when mutual competition influences the adsorption processes. Selectivity for the adsorption of lanthanide ions increases in the order $\mathrm{La}^{3+}<\mathrm{Nd}^{3+}<\mathrm{Lu}^{3+}<\mathrm{Eu}^{3+}<\mathrm{Dy}^{3+}$. This again confirms the potential use of this material for the separation of rare earths by means of ion-exchange column chromatography.

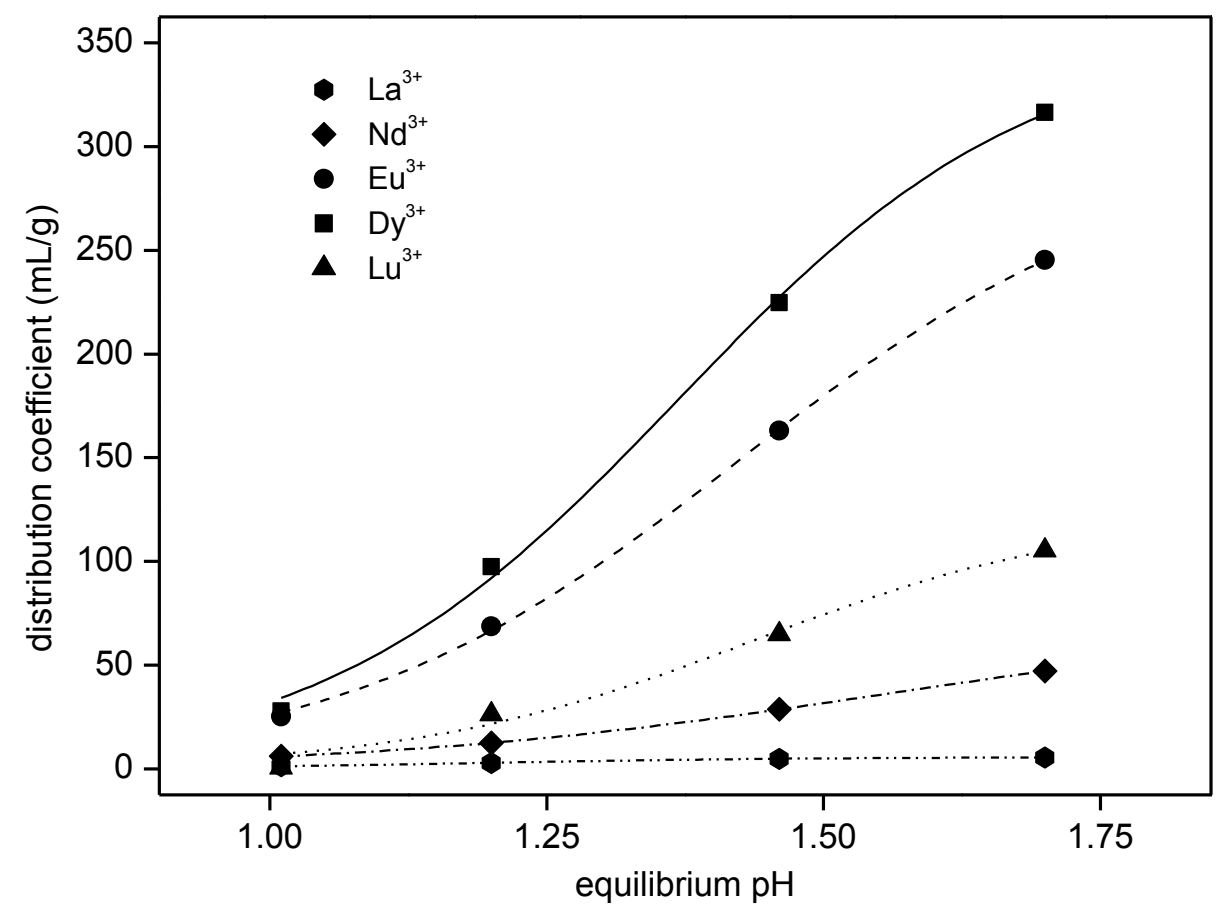

Figure 11: Distribution coefficients for adsorption of different lanthanide ions from a multi-element solution with DTPA-chitosan-silica as a function of equilibrium $\mathrm{pH}$.

A simpler system, the $\mathrm{Nd} / \mathrm{Dy}$ couple, was investigated to quantify the selectivity. Neodymium and dysprosium are the two rare earths that the US Department of Energy (DOE) ranked highest in importance to both clean energy and supply risk. ${ }^{52}$ As both elements occur in $\mathrm{NdFeB}$ magnets, the separation of neodymium and dysprosium is very relevant. A binary mixture of $\mathrm{Nd}^{3+}$ and 
$\mathrm{Dy}^{3+}$ was prepared $\left(c_{\mathrm{Ln}}{ }^{3+}=0.52 \mathrm{mmol} \mathrm{L}^{-1}\right)$ and subjected to adsorption with EDTA- and DTPAchitosan-silica. To quantify the difference in adsorption amount of both ions, an enrichment factor was calculated, defined as the ratio of $\mathrm{Dy}^{3+}$ to $\mathrm{Nd}^{3+}$ present at equilibrium onto the adsorbents and $\mathrm{Dy}^{3+}$ to $\mathrm{Nd}^{3+}$ initially present in the aqueous solution:

$$
\text { enrichment factor }=\frac{\left(\frac{\left[D y^{3+}\right]}{\left[N d^{3+}\right]}\right)_{a d s, e q}}{\left(\frac{\left[D y^{3+}\right]}{\left[N d^{3+}\right]}\right)_{a q, i n}}
$$

The calculated enrichment factors as a function of equilibrium $\mathrm{pH}$ are visualized for both EDTAchitosan-silica and DTPA-chitosan-silica in Figure 12. The most important conclusion is that selectivity is high for both materials. For both EDTA-chitosan-silica and DTPA-chitosan-silica the enrichment factor is 2 at $\mathrm{pH} 2.00$, which means that twice as much dysprosium is adsorbed in comparison with neodymium. This is a good value with respect to the application of these materials as resins for column chromatography. Moreover, by decreasing the $\mathrm{pH}$, the number of available sorption sites becomes smaller and competition increases. The differences in affinity are exploited and selectivity increases. While the maximum selectivity is reached for EDTAchitosan-silica at $\mathrm{pH} 1.50$ (still around a value of 2), for DTPA-chitosan-silica the number of adsorbed $\mathrm{Nd}^{3+}$ ions decreases faster than the number of adsorbed $\mathrm{Dy}^{3+}$ ions with decreasing $\mathrm{pH}$. An enrichment factor higher than 3 is reached for DTPA-chitosan-silica at $\mathrm{pH} 1.00$. The high selectivity at low $\mathrm{pH}$ is remarkable and advantageous for the selective recovery of rare earths from leaching solutions, which are characterized by low $\mathrm{pH}$ values. The figure does not show the stripping effect below $\mathrm{pH} 1$ which causes the selectivity to drop for both materials. 


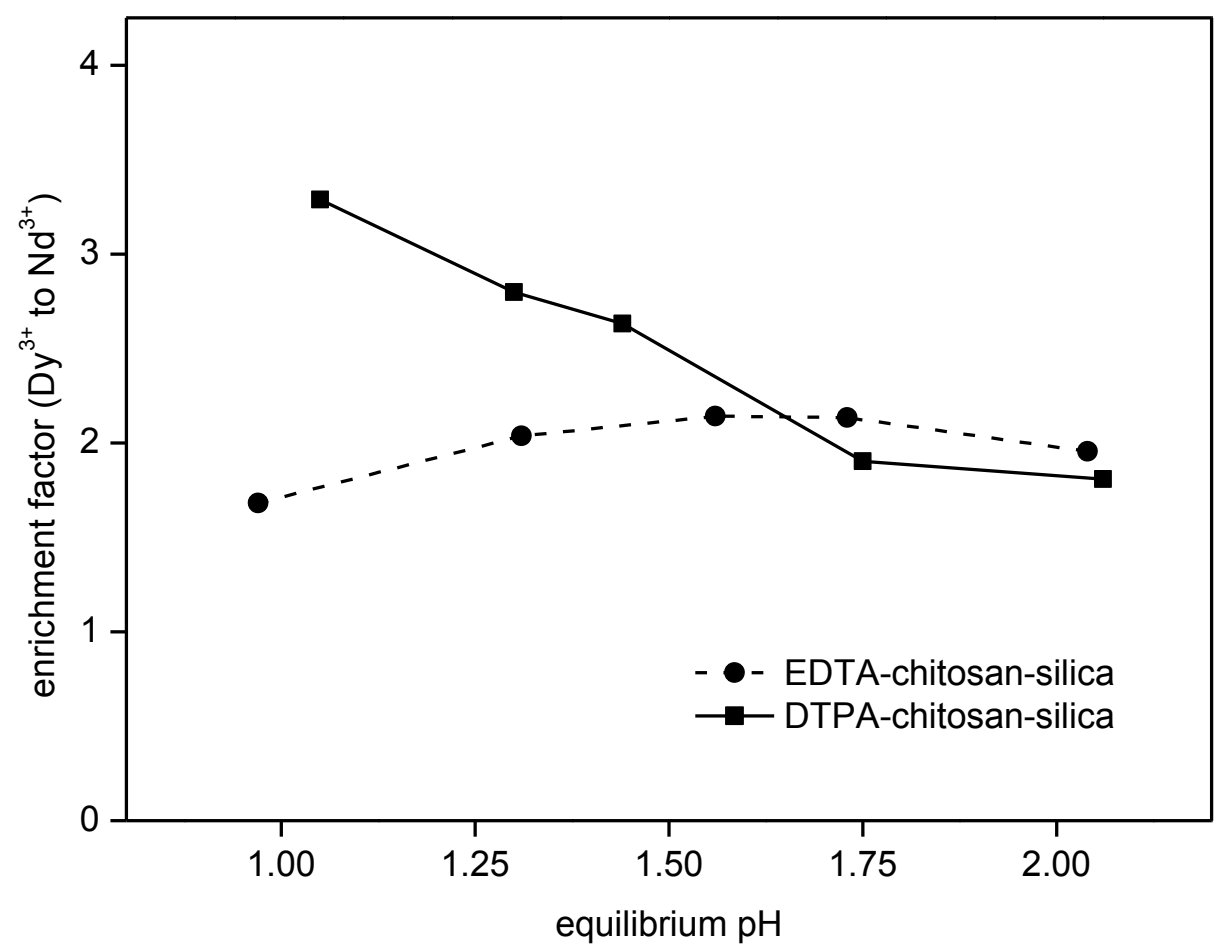

Figure 12: Enrichment factors for adsorption of Dy ${ }^{3+}$ in comparison with $\mathrm{Nd}^{3+}$ for EDTA-chitosan-silica and DTPA-chitosan-silica as a function of equilibrium $\mathrm{pH}$.

\section{Separation}

After investigation of the selectivity of DTPA-chitosan-silica for the industrially relevant elements neodymium and dysprosium, an actual separation of the ions of these two metals was performed by using the functionalized hybrid material as a resin in a chromatography column, under medium-pressure conditions ( $\leq 10 \mathrm{bar}$ ). After conditioning the column to a $\mathrm{pH}$ of 1.50 , the sample, $10 \mathrm{~mL}$ of an aqueous $1: 1 \mathrm{Nd}^{3+} / \mathrm{Dy}^{3+}$ mixture, was added on the top of the column, followed by an additional $15 \mathrm{~mL}$ of aqueous $\mathrm{HNO}_{3}$ of $\mathrm{pH} 1.50$. Breakthrough of neodymium was initiated by elution with, in succession, $50 \mathrm{~mL}$ of aqueous $\mathrm{HNO}_{3}$ of $\mathrm{pH} 1.25$ and $50 \mathrm{~mL}$ of aqueous $\mathrm{HNO}_{3}$ of $\mathrm{pH} 1.00$. Eventually, stripping was performed by elution with $50 \mathrm{~mL}$ of $1.0 \mathrm{M}$ $\mathrm{HNO}_{3}$. In Figure 13, it can be observed that neodymium and dysprosium are quasi-quantitatively separated from each other in one simple chromatography cycle. It is observed that a $\mathrm{pH}$ of 1.00 
was necessary to make neodymium break through the column. At this $\mathrm{pH}$ however, dysprosium stayed complexed with the functional groups immobilized on the column packing. Hence, both elements could be collected in different fractions and separation was thus achieved. By stripping of dysprosium with 1.0 $\mathrm{M} \mathrm{HNO}_{3}$, residual amounts of bonded neodymium co-eluted from the column. These ions can be considered as contamination. The corresponding fractions could eventually be subjected to one or more extra chromatographic cycles to get purer elements. Further, the resin could, in principle, be reused for many other separation experiments, as shown in the reusability studies, but an in-depth study of relevant separations is considered more relevant after development of high-performance 3D-shaped functional materials.

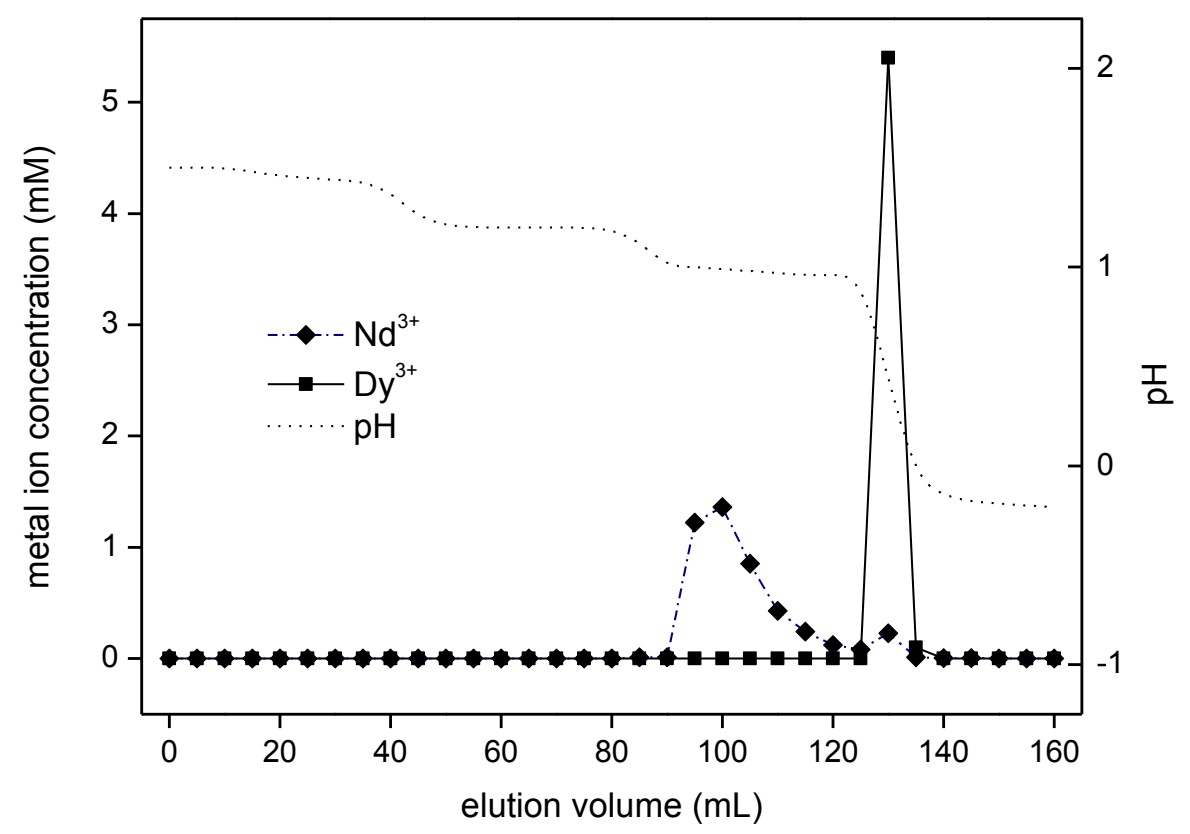

Figure 13: Chromatogram of the separation of a $\mathrm{Nd}^{3+} / \mathrm{Dy}^{3+}$ mixture $\left(\mathrm{CLn}^{3+}=2.0 \mathrm{mM}\right)$ by elution with an aqueous $\mathrm{HNO}_{3}$ solution. 


\section{CONCLUSIONS}

EDTA- and DTPA-chitosan-silica were synthesized via a sol-gel hybridization reaction and fully characterized. With different techniques, it was calculated that, on average, $30 \mathrm{wt} \%$ of the biopolymer consisted of functionalized organic material and $70 \mathrm{wt} \%$ of silica. This composition was reflected in the porous and rigid character of the particles that could thus be used as carrier in a chromatography column. Comparison of the luminescence decay times of the europium(III) coordinated complexes in water and in heavy water allowed the determination of the hydration number, which was 3 for EDTA- and 1 for DTPA-chitosan-silica, resulting in a coordination number of 8 for europium(III) in both materials. During adsorption experiments, equilibrium conditions were reached after three hours. The adsorption capacity of DTPA-chitosan-silica was shown to be higher than that of EDTA-chitosan-silica. Maximum adsorption was reached for $\mathrm{pH}$ 4 and above. Functionalized particles were fully stripped by treatment with $1 \mathrm{M} \mathrm{HCl}$ solution. The adsorption efficiency dropped by about $15 \%$ after one stripping step and remained rather constant in the following reusability cycles. DTPA-chitosan-silica showed a higher selectivity than EDTA-chitosan-silica towards adsorption of dysprosium(III) in comparison with neodymium(III). This selectivity arises from mutual differences in affinity for the different lanthanide ions. These findings clearly show the potential use of these hybrid materials as resins for the separation of rare earths by means of ion-exchange column chromatography. Thanks to the structural advantages of the incorporated silica, these materials are very suited for use as a stationary phase in a column resin. This was shown in the last experiment, in which also the metal-separating efficiency of this material was proven to be sufficient for the mutual separation neodymium and dysprosium in one simple chromatography cycle. 


\section{Acknowledgements}

The authors thank FWO Flanders, VITO and KU Leuven (IOF-KP RARE ${ }^{3}$ ) for financial support. SEM pictures were made by Raymond Kemps (VITO). BET and TGA analyses were performed by Anne-Marie De Wilde (VITO). CHN analyses were carried out by Dirk Henot (KU Leuven). David Dupont and Bieke Onghena (KU Leuven) are acknowledged for useful discussions and suggestions about the experimental work.

\section{Electronic Supporting Information}

Electronic supplementary information (ESI) available: TGA results, ICP data and CHN values. 


\section{References}

1. M. Fomina and G. M. Gadd, Bioresour. Technol., 2014, 160, 3-14.

2. J. Wang and C. Chen, Biotechnol Adv, 2009, 27, 195-226.

3. S. Babel and T. A. Kurniawan, J. Hazard. Mater., 2003, B97, 219-243.

4. S. E. Bailey, T. J. Olin, R. M. Bricka and D. D. Adrian, Water Res., 1999, 33, 2469-2479.

5. G. Crini, Bioresour. Technol., 2006, 97, 1061-1085.

6. E. Guibal, Sep. Purif. Technol., 2004, 38, 43-74.

7. H. Sashiwa and S.-i. Aiba, Prog. Polym. Sci., 2004, 29, 887-908.

8. E. Repo, J. K. Warchol, T. A. Kurniawan and M. E. T. Sillanpää, Chem. Eng. J., 2010, $161,73-82$.

9. E. Guibal, A. Larkin, T. Vincent and J. M. Tobin, Ind. Eng. Chem. Res., 1999, 38, 40114022.

10. K. Inoue, Y. Baba and K. Yoshizuka, Bull. Chem. Soc. Jpn., 1993, 66, 2915-2921.

11. K. Inoue, T. Yamaguchi, M. Iwasaki, K. Ohto and K. Yoshizuka, Sep. Sci. Technol., 1995, 30, 2477-2489.

12. E. Repo, R. Koivula, R. Harjula and M. Sillanpää, Desalination, 2013, 321, 93-102.

13. P. S. Barber, S. P. Kelley, C. S. Griggs, S. Wallace and R. D. Rogers, Green Chem., 2014, 16, 1828-1836.

14. E. Guibal, C. Milot and J. M. Tobin, Ind. Eng. Chem. Res., 1998, 37, 1454-1463.

15. M. Ruiz, A. M. Sastre and E. Guibal, React. Funct. Polym., 2000, 45, 155-173.

16. A. J. Varma, S. V. Deshpande and J. F. Kennedy, Carbohydr. Polym., 2004, 55, 77-93.

17. C. Gerente, V. K. C. Lee, P. L. Cloirec and G. McKay, Crit. Rev. Environ. Sci. Technol., 2007, 37, 41-127.

18. R. B. N. Baig, M. N. Nadagouda and R. S. Varma, Green Chem., 2014, 16, 2122-2127.

19. R. B. N. Baig and R. S. Varma, Green Chem., 2013, 15, 1839. 
20. E. Guibal, Prog. Polym. Sci., 2005, 30, 71-109.

21. J. J. E. Hardy, S. Hubert, D. J. Macquarrie and A. J. Wilson, Green Chem., 2004, 6, 5356.

22. B. C. E. Makhubela, A. Jardine and G. S. Smith, Green Chem., 2012, 14, 338-347.

23. Y. Liu, X. Cao, R. Hua, Y. Wang, Y. Liu, C. Pang and Y. Wang, Hydrometallurgy, 2010, $104,150-155$.

24. S. Madala, S. V. Nadavala, S. Vudagandla, V. M. Boddu and K. Abburi, Arab. J. Chem., 2013, http://dx.doi.org/10.1016/j.arabjc.2013.1007.1017.

25. W. S. W. Ngah and S. Fatinathan, Chem. Eng. J., 2008, 143, 62-72.

26. H. Y. Zhu, Y. Q. Fu, R. Jiang, J. Yao, L. Xiao and G. M. Zeng, Bioresour. Technol., 2012, 105, 24-30.

27. C. Liu, N. Naismith and J. Economy, J. Chromatogr. A, 2004, 1036, 113-118.

28. D. H. Reddy and S. M. Lee, Adv. Colloid Interface Sci., 2013, 201-202, 68-93.

29. H. Cui, J. Chen, H. Yang, W. Wang, Y. Liu, D. Zou, W. Liu and G. Men, Chem. Eng. J., $2013,232,372-379$.

30. E. Repo, L. Malinen, R. Koivula, R. Harjula and M. Sillanpaa, J. Hazard. Mater., 2011, $187,122-132$.

31. P. Dhawade and R. Jagtap, Pelagia Research Library, 2012, 3, 589-601.

32. M. R. Gandhi and S. Meenakshi, Int. J. Biol. Macromol., 2012, 50, 650-657.

33. S. S. Rashidova, D. S. Shakarova, O. N. Ruzimuradov, D. T. Satubaldieva, S. V. Zalyalieva, O. A. Shpigun, V. P. Varlamov and B. D. Kabulov, J. Chromatogr. B, 2004, $800,49-53$.

34. E. Repo, J. K. Warchol, A. Bhatnagar and M. E. T. Sillanpää, J. Colloid Interface Sci., $2011,358,261-267$.

35. T. Witoon and M. Chareonpanich, Ceram. Int., 2012, 38, 5999-6007. 
36. F. Li, X. M. Li and S. S. Zhang, J. Chromatogr. A, 2006, 1129, 223-230.

37. V. K. Gupta and Suhas, J Environ Manage, 2009, 90, 2313-2342.

38. K. Binnemans, Y. Pontikes, P. T. Jones, T. Van Gerven and B. Blanpain, presented in part at the Proceedings of the Third International Slag Valorisation Symposium, Leuven, Belgium, 19-20 March 2013.

39. K. Binnemans, P. T. Jones, B. Blanpain, T. Van Gerven, Y. Yang, A. Walton and M. Buchert, J. Clean. Prod., 2013, 51, 1-22.

40. K. Binnemans and P. T. Jones, J. Rare Earth, 2014, 32, 195-200.

41. M. Tanaka, T. Oki, K. Koyama, H. Narita and T. Oishi, in Handbook on the Physics and Chemistry of Rare Earths, 2013, vol. 43, ch. 255, pp. 159-211.

42. F. Wang, J. Zhao, F. Pan, H. Zhou, X. Yang, W. Li and H. Liu, Ind. Eng. Chem. Res., $2013,52,3453-3461$.

43. K. Inoue and S. Alam, JOM, 2013, 65, 1341-1347.

44. J. Roosen and K. Binnemans, J. Mater. Chem. A, 2014, 2, 1530.

45. C. F. Brinker and G. W. Scherer, Sol-Gel Science. The Physics and Chemistry of Sol-Gel Processing., Academic Press, San Diego, California, 1990.

46. K. Binnemans and C. Gorller-Walrand, J. Rare Earth, 1996, 14, 173-180.

47. R. M. Supkowski and W. D. J. Horrocks, Inorg. Chim. Acta, 2002, 340, 44-48.

48. R. Smith and A. Martell, Critical Stability Constants, Plenum Press, New York, 1976.

49. G. Alberti, V. Amendola, M. Pesavento and R. Biesuz, Coord. Chem. Rev., 2012, 256, $28-45$.

50. K. Y. Foo and B. H. Hameed, Chem. Eng. J., 2010, 156, 2-10.

51. R. H. Byrne and L. Biqiong, Geochim. Cosmochim. Acta, 1995, 59, 4575-4589.

52. U. S. Department of Energy, Critical Materials Strategy, 2010. 